\title{
Dispatching Vehicles Considering Multi-lifts of Quay Cranes
}

\author{
Vu Duc Nguyen \\ Department of Industrial Engineering, Pusan National University \\ 30 Jangjeon-dong, Geumjeong-gu, Busan 609-735, South Korea \\ Tel: +82-51-510-2419, Fax: +82-51-512-7603, E-mail: duc.ise @pusan.ac.kr \\ Kap Hwan Kim ${ }^{\dagger}$ \\ Department of Industrial Engineering, Pusan National University \\ 30 Jangjeon-dong, Geumjeong-gu, Busan 609-735, South Korea \\ Tel: +82-51-510-2419, Fax: +82-51-512-7603, E-mail: kapkim@pusan.ac.kr
}

Received December 21, 2009; Revised February 24, 2010; Accepted March 2, 2010

\begin{abstract}
To improve the ship operation in automated container terminals, it is important to schedule different types of handling equipment to operate synchronously. For example, a vehicle with container receiving and lifting capabilities is used to transport containers from a storage yard to a vessel and vice versa, while a triple quay crane (QC) can handle up to three $40-\mathrm{ft}$ containers simultaneously. This paper discusses the manner in which vehicles should be assigned to containers to support such multi-lifts of QCs by using information about the locations and times of deliveries. A mixed-integer programming model is introduced to optimally assign delivery tasks to vehicles. This model considers the constraint imposed by the limited buffer space under each QC. A procedure for converting buffer-space constraints into time window constraints and a heuristic algorithm for overcoming the excessive computational time required for solving the mathematical model are suggested. A numerical experiment is conducted to compare the objective values and computational times of the heuristic algorithm with those of the optimizing method to evaluate the performance of the heuristic algorithm.
\end{abstract}

Keywords: Vehicles, Container Terminal, Dispatching, Time Window, Multi-lifts

\section{INTRODUCTION}

The transport of containers via seaport container terminals has rapidly increased during the last decade. An increasing number of countries have recognized the resulting challenges and opportunities for investment. In particular, the governments of Hong Kong, Singapore, Korea, and China have invested large amounts of money to increase the capacities of their container terminals. Therefore, the competition between seaports has become much more severe.

One of the most important issues facing container terminals is how to reduce the time that is needed for ship operations. The ship operation process consists of unloading and loading operations, as well as the transport of containers between vessels and storage blocks. In this paper, we consider an automated port container terminal in which three main types of equipment, i.e.,
QCs, vehicles, and yard cranes (YCs), are used for ship operations. Nowadays, the development of handling equipment in container terminals is improving the ship operation. This paper considers the multi-lifts of QCs, which are capable of transferring up to six 20 -ft containers or three $40-\mathrm{ft}$ containers. The triple lift of a QC is shown in Figure 1. Recently, some modern container terminals have applied the multi-lifts of QCs to improve the ship operation. However, with such multi-lifts, since an increasing number of containers will be transferred by QCs, it is necessary to deal with the problem of how to distribute and supply containers to eliminate delays and make the QC operation efficient.

During an unloading operation, up to three $40-\mathrm{ft}$ containers are picked up by a triple $\mathrm{QC}$ and put down in the buffer space under the QC in an apron. Then, vehicles pick up and deliver the containers to the storage yard. In the yard, the vehicle releases a container in the

$\dagger$ : Corresponding Author 
buffer space at a transfer point (TP) of the yard. A YC picks up and stacks it in an empty slot in a bay. A loading operation is performed in the reverse order of the unloading process. Figure 2 illustrates the layout of an automated port container terminal that has buffer space under the QCs and TPs in an apron and yard, respectively.

In the field of manufacturing scheduling (Morikawa and Takahashi, 2009), the dispatching of vehicles has been treated as an important issue. In previous researches on vehicle dispatching methods, it was assumed that pickup calls were issued randomly and that the sequence of the calls could not be known in advance. Thus, the dispatching decision was made after a pickup call was issued or when a vehicle became free from a previous delivery task (Egbelu, 1987; Bilge and Ulusoy, 1995; Kim et al., 1999; Lim et al., 2003; Grunow et al., 2004, 2006; Briskorn et al., 2006; Correa et al., 2007). However, in practice, before a vessel arrives at a port, a work schedule for the QCs is created on the basis of a stowage plan, which is used as a guideline for unloading and loading operations. Then, a sequence list of unloading and loading operations for individual containers is made by using the work schedule. When the vessel actually arrives at its berth, ship operations are carried out in the same order as specified in the sequence list. Thus, it can be said that the delivery tasks of vehicles are known in advance and that their sequence is also predetermined. Bish et al. (2005) introduced a vehicle-dispatching problem in which a detailed sequence specifying the order of the containers that are to be discharged/loaded onto the ship is given. They easily developed implementable heuristic algorithms and identified both the absolute and asymptotic worst-case performance ratios of these heuristics.

In this paper, dispatching can be defined as the assignment of vehicles to delivery tasks. Specific buffer spaces in aprons and yards are considered, within which QCs, YCs, and vehicles can release containers. We will assume that a QC performs triple lifts in which three 40$\mathrm{ft}$ containers are handled simultaneously. QCs or YCs can pick up (put down) containers directly from (onto) a vehicle. However, because a vehicle is also capable of lifting a container from the ground by itself, a QC can put three $40-\mathrm{ft}$ containers in the buffer space if sufficient buffer space is available (at least 3 empty buffer spaces are required). Therefore, in relation to releasing containers in the buffer space, a vehicle or QC must wait when there is insufficient buffer space. The number of containers that can be transferred by QCs capable of performing triple lifts is much larger than that transferred by QCs capable of performing single operations. Therefore, in order to make ship operations efficient, it is essential to determine the manner in which vehicles must be dispatched to support QC operations to minimize the delay times of QCs, as well as the travel times of vehicles.

Together with the development of equipment techniques, we introduce more efficient and functional hand- ling equipment such as automated lifting vehicles (ALVs) with the capacity of picking up containers from the floor. However, a few previous studies have examined ALVs. Through a simulation study, Van Der Meer (2000) evaluated various dispatching rules, including rules using pre-arrival information, for ALVs in container terminals. Vis and Harika (2004) and Yang et al. (2004) compared the performance of two types of automated vehicles, namely, AGVs and ALVs, by a simulation study.

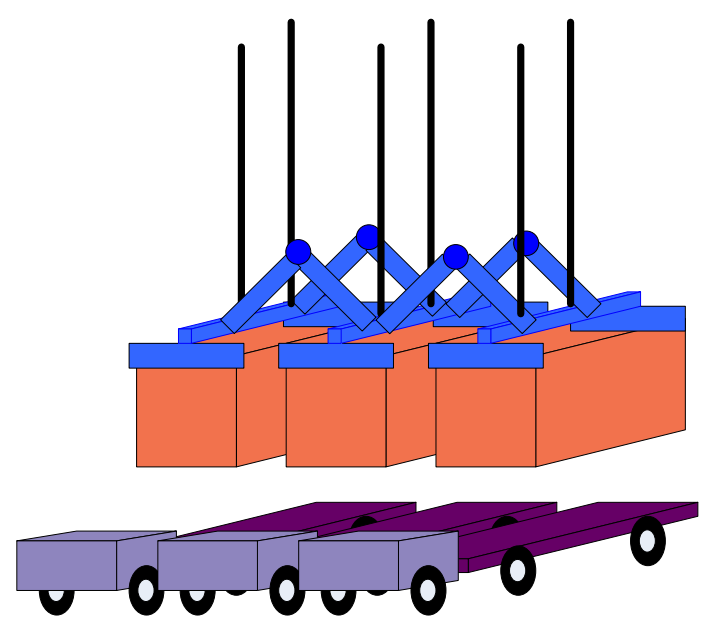

Figure 1. QC with triple lift capability in container terminal.



Figure 2. Layout of an automated container terminal.

Recently, Nguyen and Kim (2009, 2010) developed a mathematical formulation of the dispatching problem for ALVs. They suggested a heuristic algorithm and compared the solutions of this heuristic algorithm with optimal solutions. Unlike their study, this study addre- 
sses the vehicle-dispatching problem considering the multi-lifts of QCs.

This paper is organized as follows. The ship operations with multi-lifts of QCs in automated container terminals are introduced in section 2. Section 3 suggests a mixed integer programming model for vehicle dispatching that considers the multi-lifts of QCs. Section 4 proposes a procedure for converting buffer constraints into time window constraints and a heuristic algorithm for solving the dispatching problem that uses these time window constraints. Section 5 shows a numerical experiment to compare the heuristic algorithm with an optimizing method and evaluate the performance of the heuristic algorithm. Finally, section 6 concludes this study and discusses further studies.

\section{SHIP OPERATION WITH MULTI-LIFTS OF QUAY CRANES}

Before a vessel arrives at a port container terminal, a sequence list of the unloading and loading operations for individual containers is prepared. The stowing plan is devised to allow tandem and triple lifts. When the vessel actually arrives, ship operations are usually performed on the basis of the sequence list of loading and discharging.

At the quayside, an unloading operation cycle by a QC ends with the release of containers onto the buffer space at the QC in an apron, while a loading operation cycle begins with the pickup of containers from the buffer space at the QC in an apron. In this study, the QC operations are triple lifts, as shown in Figure 3c. In this figure, there are six possible combinations of triple lifts, with up to three $40-\mathrm{ft}$ containers or six 20 -ft containers. For tandem lifts, two $40-\mathrm{ft}$ containers or four 20 -ft containers can be lifted simultaneously Figure $3 \mathrm{~b}$. While single lifts can only handle one 40 - $\mathrm{ft}$ container or two $20-\mathrm{ft}$ containers Figure $3 \mathrm{a}$. The ability of tandem or triple QCs to handle a larger number of containers than single QCs is expected to enhance ship operations in container terminals.

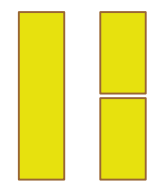

(a) Single lifts

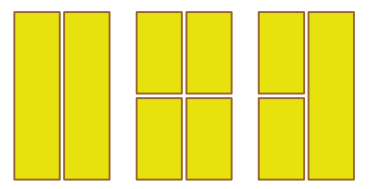

(b) Tandem lifts



(c) Triple lifts
Figure 3. Types of operations of QCs.
Table 1 shows an example of a sequence list for applying QC 1 to ship operations, to perform different types of QC operations. As shown in column 3, QC 1 is used for tandem and triple lifts. Table 2 explains the progress of the operations performed by QC 1 as it follows the sequence list shown in Table 1.

Table 2 lists the events and their notations in the ship operations of QC 1 . Table 2 assumes that the operation of QC 1 is not delayed because of the late arrival of vehicles or full occupancy of the buffer space. With

Table 1. Example of a sequence list of different types of operation of QC 01.

\begin{tabular}{|c|c|c|c|c|c|c|}
\hline \multicolumn{7}{|c|}{ QC 01 } \\
\hline $\begin{array}{c}\text { Task } \\
\text { sequence }\end{array}$ & Type $^{\dagger}$ & $\begin{array}{l}\text { No. of } 40-\mathrm{ft} \\
\text { containers }\end{array}$ & $\begin{array}{c}\text { Ship } \\
\text { location }^{\neq}\end{array}$ & $\begin{array}{c}\text { Yard } \\
\text { location }\end{array}$ & $\begin{array}{l}\text { Operation } \\
\text { cycle time }\end{array}$ & $\begin{array}{c}\text { Earliest } \\
\text { event time }\end{array}$ \\
\hline 1 & L & 3 & $\begin{array}{c}06 / 03 / 04 \\
06 / 04 / 04 \\
06 / 05 / 0\end{array}$ & $\begin{array}{l}\mathrm{A} / 21 / 3 / 2 \\
\mathrm{~A} / 11 / 1 / 1 \\
\mathrm{~B} / 05 / 3 / 4\end{array}$ & 120 & 0 \\
\hline 2 & $\mathrm{U}$ & 2 & $\begin{array}{l}10 / 03 / 10 \\
10 / 04 / 10\end{array}$ & $\begin{array}{l}\mathrm{B} / 17 / 4 / 2 \\
\mathrm{~B} / 21 / 7 / 3\end{array}$ & 110 & 230 \\
\hline 3 & $\mathrm{~L}$ & 2 & $\begin{array}{l}18 / 05 / 06 \\
18 / 06 / 06\end{array}$ & $\begin{array}{l}\mathrm{A} / 09 / 4 / 2 \\
\mathrm{~B} / 07 / 4 / 1\end{array}$ & 120 & 230 \\
\hline 4 & U & 3 & $\begin{array}{l}26 / 04 / 08 \\
26 / 05 / 08 \\
26 / 06 / 08\end{array}$ & $\begin{array}{l}\mathrm{A} / 21 / 3 / 1 \\
\mathrm{~B} / 13 / 8 / 1 \\
\mathrm{~B} / 15 / 6 / 4\end{array}$ & 110 & 460 \\
\hline$\ldots$ & $\ldots$ & $\ldots$ & $\ldots$ & $\ldots$ & $\ldots$ & $\ldots$ \\
\hline
\end{tabular}

Table 2. Events of the QC 1 ship operation.

\begin{tabular}{|c|c|c|c|}
\hline $\begin{array}{c}\text { Event } \\
\text { se- } \\
\text { quence }\end{array}$ & Time & Notation & Description \\
\hline \multirow{2}{*}{1} & 0 & $q_{1}^{1}$ & $\begin{array}{l}\text { As the } 1^{\text {st }} \text { task, QC } 1 \text { picks up three } \\
\text { containers from the buffer at QC } 1 \\
\text { (assume that three containers were } \\
\text { ready in the buffer at QC } 1 \text { ). }\end{array}$ \\
\hline & 120 & & $\begin{array}{l}\text { QC 1 releases the three containers of } \\
\text { the } 1^{\text {st }} \text { task in the ship's bay, and picks } \\
\text { up the two containers of the } 2^{\text {nd }} \text { task } \\
\text { from the ship's bay. }\end{array}$ \\
\hline \multirow[t]{2}{*}{$\begin{array}{l}2 \\
3\end{array}$} & 230 & $\begin{array}{l}q_{2}^{1} \\
q_{3}^{1}\end{array}$ & $\begin{array}{l}\text { QC 1 releases the two containers of } \\
\text { the } 2^{\text {nd }} \text { task into the buffer (at this } \\
\text { time, sufficient buffer space must be } \\
\text { available) and picks up the two con- } \\
\text { tainers of the } 3^{\text {rd }} \text { task from the buffer } \\
\text { at QC } 1 \text { (assume that two containers } \\
\text { were ready in the buffer at QC } 1 \text { ). }\end{array}$ \\
\hline & 350 & & $\begin{array}{l}\text { QC 1 releases the two containers of } \\
\text { the } 3^{\text {rd }} \text { task in the ship's bay, and } \\
\text { picks up the three containers of the } 4^{\text {th }} \\
\text { task from the ship's bay. }\end{array}$ \\
\hline 4 & 460 & $q_{4}^{1}$ & $\begin{array}{l}\text { QC 1 releases the three containers of } \\
\text { the } 4^{\text {th }} \text { task into the buffer at QC } 1 \text { (at } \\
\text { this time, sufficient buffer space must } \\
\text { be available). }\end{array}$ \\
\hline
\end{tabular}


regard to the loading operation of a $\mathrm{QC}$, which is to be performed without delay, the assigned vehicles must put down the loading containers into a specific buffer space at the QC in an apron before the QC can begin to pick them up. For an unloading operation, the buffer space must be available for the inbound containers at the QC apron or the assigned vehicles must be ready to receive the inbound containers. It should be noted that because the QC operations considered in this study are multi-lifts, a number of containers must be ready in the buffer space for a loading operation by a QC and sufficient buffer space must be available for an unloading operation (at least 2 slots for tandem lifts and 3 slots for triple lifts are required).

\section{MODEL FORMULATION}

The dispatching problem for ALVs was formulated as a mixed integer programming (MIP) model by Nguyen and Kim (2009). However, this study addresses the vehicle-dispatching problem considering the multi-lifts of QCs. To formulate the mathematical model, some notations from Nguyen and Kim (2009) are summarized, and additional notations are defined in this section.

Let $q_{i}^{k}$ be an event representing the moment that QC $k$ transfers the containers of the $i^{\text {th }}$ operation (of QC $k$ ). When the $i^{\text {th }}$ operation of QC $k$ is a loading operation, event $q_{i}^{k}$ corresponds to the pickup of the containers of the $i^{\text {th }}$ operation of QC $k$ from the buffer at QC $k$. When the $i^{\text {th }}$ operation of QC $k$ is an unloading operation, it corresponds to the release of the containers of the $i^{\text {th }}$ operation by QC $k$ into the buffer at QC $k$. The event time of $q_{i}^{k}$ is denoted by $y_{i}^{k}$, which is a decision variable.

Let $a_{i e}^{k}$ also be an event representing the moment that a vehicle transfers the $e^{\text {th }}$ container of the $i^{\text {th }}$ operation of QC $k$. When the $i^{\text {th }}$ operation of QC $k$ is a loading operation, event $a_{i e}^{k}$ corresponds to the release of the $e^{\text {th }}$ container of the $i^{\text {th }}$ operation by vehicle into the buffer at QC $k$. When the $i^{\text {th }}$ operation of QC $k$ is an unloading operation, it corresponds to the pickup of the eth container of the $i^{\text {th }}$ operation by a vehicle from the buffer at QC $k$. The event time of $a_{i e}^{k}$ is denoted by $z_{i e}^{k}$.

Let $s_{i}^{k}$ be the earliest possible event time of $q_{i}^{k}$.

The additional notations are summarized as follows: $a_{0 j}^{o}=$ The starting event of vehicle $j, j \in V$.

$a_{0 j}^{F}=$ The stopping event of vehicle $j, j \in V$.

$m_{k}=$ The number of operations for QC $k$.

$n_{i}=$ The number of $40-\mathrm{ft}$ containers of operation i, $n_{i}=1$, $\cdots, 3$.

$1\left(a_{i e}^{k}\right)=$ The location where event $a_{i e}^{k}$ occurs. $l\left(a_{0 j}^{o}\right)$ represents the initial location of vehicle $j . l\left(a_{i e}^{k}\right)$ represents the position where the $e^{\text {th }}$ container of the $i^{\text {th }}$ operation of QC $k$ is to be transferred. $l\left(a_{0 j}^{F}\right)$ represents the location where a vehicle completes its final delivery task.

$t_{k i e}^{l i f}=$ The pure travel time from $l\left(a_{i e}^{k}\right)$ to $l\left(a_{j f}^{l}\right)$.

$c_{k i e}^{l i f}=$ The time required for a vehicle to be ready for $a_{j f}^{l}$ after it experiences $a_{i e}^{k}$. For example, if both $a_{i e}^{k}$ and $a_{j f}^{l}$ are related to loading operations, then the starting event for evaluating $c_{\text {kie }}^{\text {lif }}$ is the release of the $e^{\text {th }}$ container of the $i^{\text {th }}$ operation by a vehicle. $c_{\text {kie }}^{\text {lif }}$ includes the travel time from an apron to the location of the next container (the $f^{\text {th }}$ container of the $j^{\text {th }}$ operation of QC $l$ ) in the marshalling yard, the pickup time of the container by vehicle, and the travel time of the vehicle to QC $l$.

$b_{k}=$ The capacity of the buffer space under QC $k$.

$M=\mathrm{A}$ large positive number.

$u_{i}^{k t}=\left\{\begin{array}{l}n_{i}, t \geq y_{i}^{k} \\ 0, t<y_{i}^{k}\end{array}\right.$, for $i \in U^{k} \cup L^{k}$ and $k \in K$.

$u_{i e}^{k t}=\left\{\begin{array}{l}1, t \geq z_{i e}^{k} \\ 0, t<z_{i e}^{k}\end{array}\right.$, for $i \in U^{k} \cup L^{k}, k \in K$ and $e=1, \cdots, n_{i}$.

$V=$ The set of vehicles.

$K=$ The set of QCs.

$S=$ The set of $a_{0 j}^{o} \quad \mathrm{~s}, j \in V$.

$D=$ The set of $a_{0 j}^{F} \quad \mathrm{~s}, j \in V$.

$L^{k}=$ The set of loading tasks, for QC $k$.

$U^{k}=$ The set of unloading tasks, for QC $k$.

$T=$ The set of $a_{i e}^{k} \mathrm{~s}$, for $i=1, \cdots, m_{k} ; e=1, \cdots, n_{i}$; and $k \in K$.

The problem in this paper is addressed as a static scheduling problem, where vehicles must be assigned to complete all the tasks in a known set under a limited buffer capacity. A feasible dispatching decision is made as a one-to-one assignment between all the events in $S \cup T$ and those in $D \cup T$.

Let $K^{\prime}=\{O\} \cup K, K^{\prime \prime}=\{F\} \cup K$, and $x_{k i e}^{l i f}$ be a decision variable that becomes 1 if $a_{i e}^{k}$ is assigned to $a_{j f}^{l}$, for $k \in K^{\prime}$ and $l \in K$ '. For $k$ and $l \in K$, the assignment of $a_{i e}^{k}$ to $a_{j f}^{l}$ implies that the vehicle, which have just delivered the $e^{\text {th }}$ container of the $i^{\text {th }}$ operation of QC $k$, is scheduled to deliver the $f^{\text {th }}$ container of the $j^{\text {th }}$ operation of QC $l$.

Let $\alpha$ be the travel cost per unit time of a vehicle, and $\beta$ be the penalty cost per unit time for a delay in the completion time. It is assumed that $\alpha<<\beta$. Further, let $m_{O}$ and $m_{F}$ be equal to $|V|$. Then, the dispatching problem can be formulated as follows.

Minimize 


$$
\alpha \sum_{k \in K^{\prime}} \sum_{i=1}^{m_{k}} \sum_{e=1}^{n_{i}} \sum_{l \in K^{\prime \prime}} \sum_{j=1}^{m_{l}} \sum_{f=1}^{n_{j}} t_{k i e}^{l i f} x_{k i e}^{l i f}+\beta \sum_{k \in K}\left(y_{m_{k}}^{k}-s_{m_{k}}^{k}\right)
$$

Subject to

$$
\begin{aligned}
& \sum_{l \in K^{\prime}} \sum_{j=1}^{m_{l}} \sum_{f=1}^{n_{j}} x_{k i e}^{l j f}=1 ; \forall k \in K^{\prime} ; \\
& i=1, \cdots, m_{k} ; e=1, \cdots, n_{j} \\
& \sum_{k \in K^{\prime}} \sum_{i=1}^{m_{k}} \sum_{e=1}^{n_{i}} x_{k i e}^{l i f}=1 ; \forall l \in K^{\prime \prime} \text {; } \\
& j=1, \cdots m_{l} ; f=1, \cdots, n_{j} \\
& z_{j f}^{l}-\left(z_{i e}^{k}+c_{k i e}^{l j f}\right) \geq M\left(x_{k i e}^{l i f}-1\right) \text {; } \\
& \forall k \in K^{\prime} ; l \in K ; i=1, \cdots, m_{k} ; j=1, \cdots, m_{l} ; \\
& e=1, \cdots, n_{i} ; f=1, \cdots, n_{j} \\
& y_{i+1}^{k}-y_{i}^{k} \geq s_{i+1}^{k}-s_{i}^{k} ; \forall k \in K ; i=1, \cdots, m_{k}-1 \\
& y_{i}^{k} \geq s_{i}^{k} ; \forall k \in K^{\prime} ; i=1, \cdots, m_{k} \\
& y_{i}^{k} \leq \min _{e}\left\{z_{i e}^{k}\right\} ; \forall k \in K^{\prime} ; i \in U^{k} ; e=1, \cdots, n_{i} \\
& y_{i}^{k} \geq \max _{e}\left\{z_{i e}^{k}\right\} ; \forall k \in K^{\prime} ; i \in L^{k} ; e=1, \cdots, n_{i} \\
& \sum_{i \in U^{k}} u_{i}^{k t}-\sum_{i \in L^{k}} u_{i}^{k t}+\sum_{i \in L^{k}} v_{i e}^{k t}-\sum_{i \in U^{k}} v_{i e}^{k t} \leq b_{k} ; \\
& \forall k \in K ; t \in\left\{y_{i, \forall i \in U^{k}}^{k}, z_{i e, \forall i \in L^{k}, e=1, \ldots, n_{i}}^{k}\right\} \\
& t-y_{i}^{k}<M \cdot u_{i}^{k t} ; \forall k \in K ; i \in U^{k} ; t \in\left\{y_{i}^{k}\right\} \\
& t-y_{i}^{k}>M \cdot\left(u_{i}^{k t}-n_{i}\right) ; \forall k \in K ; i \in L^{k} ; t \in\left\{y_{i}^{k}\right\} \\
& t-z_{i e}^{k}<M \cdot v_{i e}^{k t} ; \forall k \in K ; i \in L^{k} ; e=1, \cdots, n_{i} ; t \in\left\{z_{i e}^{k}\right\} \\
& t-z_{i e}^{k}>M \cdot\left(v_{i e}^{k t}-1\right) ; \forall k \in K ; i \in U^{k} \text {; } \\
& e=1, \cdots, n_{i} ; t \in\left\{z_{i e}^{k}\right\} \\
& u_{i}^{k t}=0 \text { or } n_{i} ; v_{i e}^{k t}=0 \text { or } 1 \text {; } \\
& \forall k \in K ; i=1, \cdots, m_{k} ; e=1, \cdots, n_{i} ; t \in\left\{y_{i}^{k}, z_{i e}^{k}\right\} \\
& x_{\text {kie }}^{\text {lif }}=0 \text { or } 1 ; \forall k \in K^{\prime} ; l \in K^{\prime \prime} \text {; } \\
& i=1, \cdots, m_{k} ; j=1, \cdots, m_{l} ; e=1, \cdots, n_{i} ; f=1, \cdots, n_{j}
\end{aligned}
$$

Note: $s_{i}^{O}=0$, for $\forall i \in V$.

From the objective function, the penalty cost per unit time is considered for a delay in the completion time. In many practical cases, early arrivals of vehicles may speed up the ship operation. Especially, in case that the handling times of QCs are uncertain, there may be some possibility that the next cycle can start earlier when the delivery of the container for the previous cycle is finished earlier. However, this paper assumes that the QC handling operation is constant and the objective is to minimize the delay times of QCs. Thus, when vehicles arrive at the transfer positions earlier than their due times, the vehicles have to wait the arrivals of the spreaders of the quay cranes. Therefore, there is no incentive for the earlier arrivals of vehicles. Objective function (1) is used to minimize the total travel time of the vehicles and the total delays in QC operations. Because $\alpha<<\beta$, the sum of the delays in the $\mathrm{QC}$ operations will be minimized first. For the same value of total delay, the total travel distance of the vehicles will be minimized. Constraints (2) and (3) force a one-to-one assignment between all the events in $S \cup T$ and those in $D \cup T$. Constraint (4) implies that two events that are served consecutively by the same vehicle must be set apart by at least the time required by a vehicle for traveling and transferring between the two events. Constraint (5) implies that two events that are served by the same QC must be set apart by at least the time required for the QC to perform all the movements between the two events. Constraint (6) implies that the actual event time is always greater than or equal to the earliest possible event time. Constraints (7) and (8) show that the containers of the $i^{\text {th }}$ operation in the buffer at QC $k$ must be available for pickup by vehicles in an unloading operation and by the QC in a loading operation, respectively. Constraint (9) indicates that the number of containers in the buffer at QC $k$ released or picked up by the QC or vehicles at time $t$ cannot exceed the capacity of the buffer. Constraint (10) ensures that $u_{i}^{k t}$ is equal to $n_{i}$ when $t>y_{i}^{k}$, which implies that $n_{i}$ containers of the $i^{\text {th }}$ operation have already been released at time $t$ by QC $k$ into the buffer at QC $k$. Constraint (11) ensures that $u_{i}^{k t}$ is equal to $n_{i}$ when $t>y_{i}^{k}$, which implies that $n_{i}$ containers of the $i^{\text {th }}$ operation have already been picked up at time $t$ by QC $k$ from the buffer at QC $k$. Constraint (12) ensures that $v_{i e}^{k t}$ is equal to 1 when $t>z_{i e}^{k}$, which implies that the $e^{\text {th }}$ container of the $i^{\text {th }}$ operation has already been released by a vehicle into the buffer at QC $k$. Constraint (13) ensures that $v_{i e}^{k t}$ is equal to 1 when $t>z_{i e}^{k}$, which implies that the $e^{\text {th }}$ container of the $i^{\text {th }}$ operation has already been picked up by a vehicle at time $t$ from the buffer at QC $k$. Constraints (14) and (15) ensure that the decision variables are restricted to binary values.

In the model formulation process, the decision variables $x_{k i e}^{\text {lif }} \mathrm{s}$ do not have an index for identifying a specific vehicle. However, a given set of $x_{k i e}^{l i f}$ values can be interpreted as an assignment of delivery tasks to specific individual vehicles as follows. Consider the single QC example in Table 1, with four operation tasks (three containers in the $1^{\text {st }}$ and $4^{\text {th }}$ operation tasks and two containers in the $2^{\text {nd }}$ and $3^{\text {rd }}$ operation tasks) and two vehicles. Suppose that a solution $\left(x_{O 01}^{111}=x_{111}^{121}=x_{121}^{122}=x_{122}^{141}\right.$ $=x_{141}^{142}=x_{142}^{F 01}=x_{O 02}^{112}=x_{112}^{113}=x_{113}^{131}=x_{131}^{132}=x_{132}^{143}=x_{143}^{F 02}=1$, and all the other $x_{\text {kie }}^{\text {lif }}=0$ ) is obtained.

A feasible solution can be represented graphically as shown in Figure 4, in which each arrow corresponds to $x_{k i e}^{l i f}$ with a value of 1 . The set $S \cup T$ includes the starting events of vehicles $(S)$ and events related to the transfer operations by vehicles $(T)$. A vehicle that started traveling from its initial location or completed a delivery for a transfer operation can be assigned to another transfer operation (event) $(T)$ or to its final destination $(D)$. Therefore, nodes in $S \cup T$ can be considered as vehicle supply sources, and nodes in $D \cup T$ as vehicle demand 


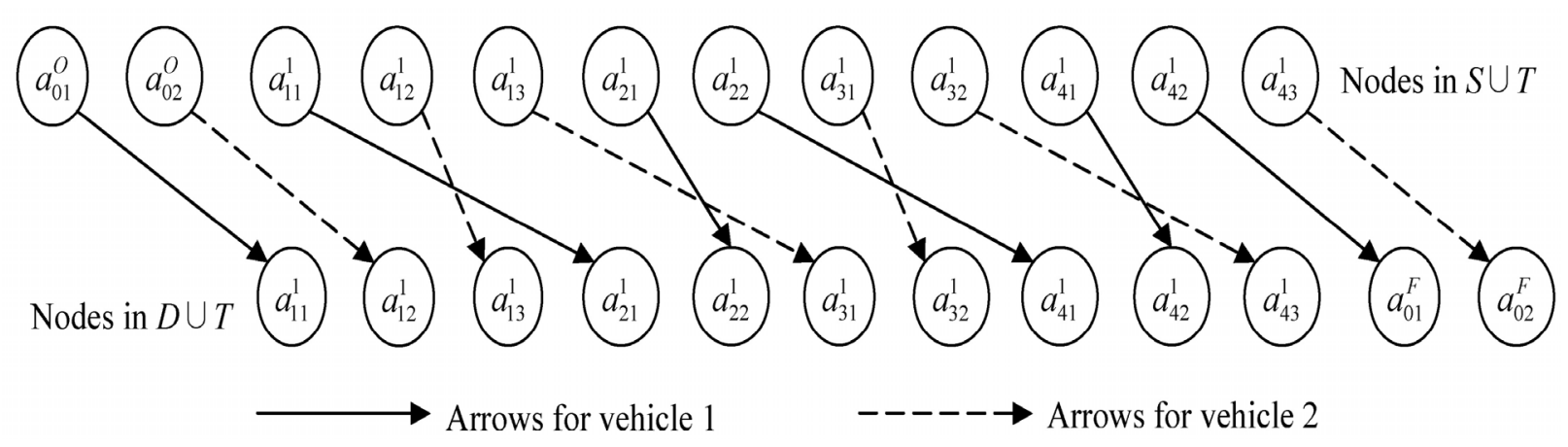

Figure 4. Graphical representation of a feasible solution.

sources. A feasible solution of the $x_{k i e}^{l i f}$ variables is a oneto-one assignment from a node in $S \cup T$ to a node in $D \cup T$.

In Figure 4, the solid arrow shows an assignment for vehicle 1, while the dashed arrow shows an assignment for vehicle 2. Node $a_{01}^{O}$ in the supply source is connected to node $a_{11}^{1}$ in the demand source by a solid arrow, which implies that vehicle 1 starts traveling empty to the pickup position of the task related to the $1^{\text {st }}$ container of the $1^{\text {st }}$ operation of QC 1 and performs the loading and travel for the task. Then, node $a_{11}^{1}$ in the supply source is connected to node $a_{21}^{1}$ in the demand source, which implies that after vehicle 1 completes the task related to the $1^{\text {st }}$ container of the $1^{\text {st }}$ operation, it performs tasks related to the two containers of the $2^{\text {nd }}$ operation. Then, it goes on to perform the tasks related to the $1^{\text {st }}$ and $2^{\text {nd }}$ containers of the $4^{\text {th }}$ operation, which implies that nodes $a_{22}^{1}$ and $a_{41}^{1}$ in the supply source are connected to nodes $a_{41}^{1}$ and $a_{42}^{1}$ in the demand source, respectively. Finally, node $a_{42}^{1}$ in the supply source is connected to node $a_{01}^{F}$ in the demand source, which can be interpreted as vehicle 1 ceases to travel after it completes all the assigned tasks. In a similar way, the solution for vehicle 2 can be interpreted as follows. Vehicle 2 delivers the $2^{\text {nd }}$ and $3^{\text {rd }}$ containers of the $1^{\text {st }}$ operation of QC 1, and then the two containers of the $3^{\text {rd }}$ operation, followed by the $3^{\text {rd }}$ container of the $4^{\text {th }}$ operation of QC 1, and finally stops traveling.

Formulations (1)-(15) are for a scheduling problem with precedence constraints and buffer constraints, which is similar to the multiple traveling salesmen problem with precedence constraints and time windows. Both these problems must be NP-hard. Thus, a heuristic algorithm is suggested in the following section to solve this problem.

\section{A HEURISTIC ALGORITHM}

As presented in the previous section, formulations (1)-(15) are addressed as a scheduling problem with precedence and buffer constraints. The heuristic algo- rithm proposed in this section consists of two steps. First, a procedure for converting the buffer constraints in the formulation into time window constraints is presented in section 4.1. Then, a heuristic algorithm for solving this scheduling problem with precedence and window constraints is suggested in section 4.2.

\subsection{Procedure for Converting buffer Constraints into time window Constraints}

A general procedure for converting buffer constraints into time window constraints in a formulation is presented in this section. At the initial stage, we suppose that the $y_{i}^{k}$ variables are equal to the $s_{i}^{k}$ variables for $i=1, \cdots, m_{k}$ and $k=1, \cdots,|K|$ and that the events are sequenced in the increasing order of $y_{i}$, for $k=1, \cdots$, $|K|$. We will denote the $j^{\text {th }}$ event in the sequence as "event $(j)$ " and the event time of event $(i)$ as $y_{i}$ for operations of a QC.

The main idea for determining the time window is to calculate the latest allowable pickup time of the inbound container for an unloading task from the buffer space and the earliest allowable release time of the outbound container for a loading task on the buffer space by a vehicle. A time axis is divided into multiple periods, each of which corresponds to a time interval between event times. Detailed explanations can be found in Nguyen and Kim (2009). However, some notations and modifications are needed to deal with the dispatching problem when considering multi-lifts by QCs.

Let $b$ be the capacity of the buffer and $m$ be the number of tasks for the QC. Denote $i$ as the index of the period in which period $i$ corresponds to the time interval between event $i-1$ and $i$. Furthermore, $n_{i}$ is the number of containers added at period $i$. Let $q_{i}$ be the number of containers in the inventory at period $i$ when the inventory plans up to the preceding event are considered. Let $W_{i}$ and $W_{i j}$ be the time window for all of the containers of task $i$ and for container $j$ of task $i$, respectively. Denote $L\left(W_{i}\right)$ and $U\left(W_{i}\right)$ as the lower and upper bounds of time window $W_{i}$, and let $L\left(W_{i j}\right)$ and $U\left(W_{i j}\right)$ be the lower and upper bounds of time window $W_{i j}$, respectively. 


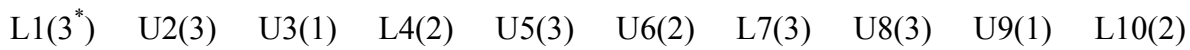

\begin{tabular}{|c|c|c|c|c|c|c|c|c|c|c|c|}
\hline Inventory Period & 1 & 2 & 3 & 4 & 5 & 6 & 7 & 8 & 9 & 10 & 11 \\
\hline \hline 5 & & & & & & & & & & & \\
\hline 4 & & & & & & & & & & & \\
\hline 3 & L1 & & & & & & & & & & \\
\hline 2 & L1 & & & & & & & & & & \\
\hline 1 & L1 & & & & & & & & & & \\
\hline
\end{tabular}

Note: ${ }^{*}$ Number of $40 \mathrm{ft}$-containers.

(a) Time window of the first task

L1(3) U2(3) $\quad$ U3(1) $\quad$ L4(2) $\quad$ U5(3) $\quad$ U6(2) $\quad$ L7(3) $\quad$ U8(3) $\quad$ U9(1) $\quad$ L10(2)

\begin{tabular}{|c|c|c|c|c|c|c|c|c|c|c|c|}
\hline $\mathrm{F}_{\text {Inventory }}$ Period & 1 & 2 & 3 & 4 & 5 & 6 & 7 & 8 & 9 & 10 & 11 \\
\hline 5 & & & & & & & & & & & \\
\hline 4 & & & 1 & $/ 2$ & & & & & & & \\
\hline 3 & L1 & & U2 & $\$ 1$ & $\mathbb{1}$ & & & & & & \\
\hline 2 & L1 & & U2 & U2 & $\$$ & & & & & & \\
\hline 1 & L1 & & U2 & U2 & U2 & & & & & & \\
\hline
\end{tabular}

(b) Time window of the first two tasks

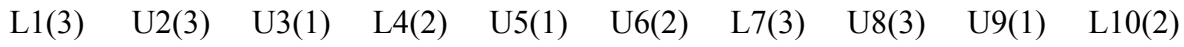

\begin{tabular}{|c|c|c|c|c|c|c|c|c|c|c|c|}
\hline $\begin{array}{ll}\text { Inventory } & \text { Period } \\
\end{array}$ & 1 & 2 & 3 & 4 & 5 & 6 & 7 & 8 & 9 & 10 & 11 \\
\hline 5 & & & & & & & & & & & \\
\hline 4 & & & & 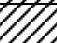 & $\mathbb{Z}$ & & & & & & \\
\hline 3 & $\mathrm{L1}$ & & U2 & U3 & & & & & & & \\
\hline 2 & $\mathrm{~L} 1$ & & U2 & U2 & U3 & 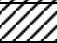 & & & & & \\
\hline 1 & L1 & & U2 & U2 & U2 & U3 & & & & & \\
\hline
\end{tabular}

(c) Time window of the first three tasks

L1(3) U2(3) $\quad$ U3(1) $\quad$ L4(2) $\quad$ U5(1) $\quad$ U6(2) $\quad$ L7(3) $\quad$ U8(3) $\quad$ U9(1) $\quad$ L10(2)

\begin{tabular}{|c|c|c|c|c|c|c|c|c|c|c|c|}
\hline Inventory Period & 1 & 2 & 3 & 4 & 5 & 6 & 7 & 8 & 9 & 10 & 11 \\
\hline \hline 5 & L4 & & L4 & L4 & & & & & & & \\
\hline 4 & L4 & & L4 & L4 & & & & & & & \\
\hline 3 & L1 & & U2 & U3 & & & & & & & \\
\hline 2 & L1 & L4 & U2 & U2 & U3 & & & & & & \\
\hline 1 & L1 & L4 & U2 & U2 & U2 & U3 & & & & & \\
\hline
\end{tabular}

(d) Time window of the first four tasks

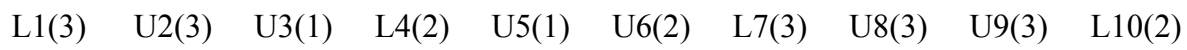

\begin{tabular}{|c|c|c|c|c|c|c|c|c|c|c|c|}
\hline Inventory Period & 1 & 2 & 3 & 4 & 5 & 6 & 7 & 8 & 9 & 10 & 11 \\
\hline \hline 5 & L4 & & L4 & L4 & L7 & L7 & L7 & & L10 & L10 & \\
\hline 4 & L4 & & L4 & L4 & L7 & L7 & L7 & L10 & L10 & L10 & \\
\hline 3 & L1 & & U2 & U3 & L7 & L7 & L7 & L10 & U8 & U9 & U9 \\
\hline 2 & L1 & L4 & U2 & U2 & U3 & U5 & U6 & U6 & U8 & U9 & U9 \\
\hline 1 & L1 & L4 & U2 & U2 & U2 & U3 & U6 & U6 & U8 & U9 & U9 \\
\hline
\end{tabular}

(e) Time window of all tasks

Time window

D. Temporary inventory at period $i$

Figure 5. Converting buffer constraints into time window constraints. 


\section{- Procedure for converting buffer constraints into time window constraints for a $Q C$}

Step 0: (Initializing) Set $y_{i}=s_{i}$ and $W_{i}=\varnothing$, for $i=1, \cdots$, $m$. And set $q_{i}=0$, for $i=1, \cdots, m$. Start $\xi=0$.

Step 1: (Next task) $\xi=\xi+1$. If $\xi>m$, then stop. Otherwise, if event $\xi$ is a loading task, then go to Step 2.1 , and if event $\xi$ is an unloading task, then go to Step 2.2.

Step 2: (Determining time windows)

Step 2.1: (Extending the lower bound for the loading task)

Start $i=\xi$.

Repeat

$q_{i}=q_{i}+n_{i}$

If $q_{i} \leq b$, then $W_{\xi}=W_{\xi} \cup\left[y_{i-1}, y_{i}\right]$ (add period $i$ to the time window for event $\xi$ ).

Otherwise, let $c=\left(n_{i}+b-q_{i}\right)$ be the remaining inventories at period $i$, the time window of $c$ containers of $n_{i}, W_{\xi e}$, is $W_{\xi e}=W_{\xi e} \cup\left[y_{i-1}\right.$, $\left.y_{i}\right], e=1, \cdots, c$.

$i=i-1$.

Until $i=0$.

Go to Step 1.

Step 2.2: (Extending the upper bound for the unloading task)

(Set tentative arrival times for loading containers)

Start $k=\xi+1$.

Repeat

If event $k$ is a loading task, then set the tentative lower bound of the time window for event $k$ to be $s=(\xi+k) / 2$. (For example, if $s=3$, then the tentative lower bound is set to be the end of period 3 ).

$k=k+1$.

Until $k>m$.

Start $i=\xi+1$.

Repeat

$r$ is the change in the number of containers at event time $i$.

$p=q_{i}+r$.

If $p \leq b$, then $W_{\xi}=W_{\xi} \cup\left[y_{i}, y_{i+1}\right]$.

Otherwise, let $c=\left(n_{i}+b-p\right)$ be the remaining inventories at period $i$, the time window of $c$ containers of $n_{i}, W_{\xi e}$, is $W_{\xi e}=W_{\xi e} \cup\left[y_{i}\right.$, $\left.y_{i+1}\right], e=1, \cdots, c$. $i=i+1$.

Until $i=m$.

Go to Step 1 .

The above procedure is illustrated in Figure 5 by using an example of ten loading and unloading operations, with a buffer capacity of five. For the first task, L1 (loading task), we extend the time window by using Step 2.1 in the procedure. Then, it is easy to obtain a time window of L1, $W_{1}=\left[0, y_{1}\right]$ (as shown in Figure 5a). For the second task, U2 (unloading task), we extend the time window by using Step 2.2 as follows: first, we estimate the period numbers corresponding to the lower bounds of the time windows for the loading tasks succeeding U2, and we denote them with an apostrophe ('). For example, we consider L4 ( $\xi=2, k=4, s=3)$, the tentative lower bound of the time window for L4, which is represented by L4', to be 3 , indicating the end of period 3. In a similar way, the tentative lower bounds for L7' and L10' are 4.5 and 6, respectively (Figure 5b). Then, we start to extend the upper bound of the time window for U2 one period at a time. For period 3, because L4' is located at the beginning of period 3 , the inventory is five, which includes three inbound containers for U2 and two outbound containers for L4. For period 4, one inbound container for U3 may arrive at the buffer; therefore, the inventory level may exceed six if the upper bound for U2 is extended to period 4. Thus, the time window for the first container of U2 becomes $W_{21}=\left[y_{2}, y_{3}\right]$. However, if one of the three inbound containers for U2 departs from the buffer, then the inventory level is five. Thus, the time window for the second container of U2 is $W_{22}=\left[y_{2}, y_{4}\right]$. For period 5, two outbound containers for $\mathrm{L} 4$ and one of the two inbound containers for U2 may depart from the buffer, and three outbound containers for L7 may arrive at the buffer; therefore, the inventory level remains five. For period 6, three inbound containers for U5 and two outbound containers for L10 may arrive at the buffer; therefore, the inventory level is ten. Thus, the time window for the third container of $\mathrm{U} 2$ becomes $W_{23}=\left[y_{2}, y_{5}\right]$.

For the third task, U3 (unloading task), the calculation is similar to that of U2. First, we estimate the tentative lower bounds for the loading tasks succeeding U3. For L4 $(\xi=3, k=4, s=3.5)$, the tentative lower bound of the time window for L4' is 3.5. In the same way, the values of $s$ for L7' and L10' are 5 and 6.5, respectively (as shown in Figure 5c). Then, we start extending the time windows one period at a time. For period 4 , the inventory becomes five: two from the arrival for U2, one for U3, and two for L4. For period 5, we have one departure of an inbound container for U2, two departures of outbound containers for L4, and three arrivals of outbound containers for L7; therefore, the inventory remains five. For period 6 , one inbound container for U2 departs from the buffer and one inbound container for U5 arrives at the buffer, leaving the inventory at five. Thus, the upper bound of the time window for U3 can be extended to the end of period 6 . For period 7 , then, if we extend the upper bound of the time window for U3 by one more period, the inventory begins to exceed the limitation of five because of the additional arrivals for $\mathrm{U} 6$ and L10. Thus, the final time window of U3 becomes $W_{3}=\left[y_{3}, y_{6}\right]$. For the fourth task, L4 (loading task), the calculation is similar to that of L1. The time window is extended in the left direction one period at a time as far as the buffer capacity allows. Thus, the time window of L4 becomes $W_{4}=\left[0, y_{4}\right]$ (as shown in Figure $5 \mathrm{~d})$. This process is repeated until the time windows for all of the tasks are determined. The time windows for the remaining tasks are, as shown in Figure $5 \mathrm{e}, W_{5}=\left[y_{5}\right.$, $\left.y_{6}\right], W_{6}=\left[y_{6}, y_{8}\right], W_{7}=\left[y_{4}, y_{7}\right], W_{8}=\left[y_{8}, y_{9}\right], W_{9}=\left[y_{9}, \infty\right]$, 
and $W_{10}=\left[y_{7}, y_{10}\right]$.

In this way, the constraints on the buffer for $z_{i e}^{k}$, (9)-(14), were converted into time window $\left(W_{i}^{k}\right)$ constraints as follows.

$$
\begin{aligned}
& z_{i e}^{k} \in W_{i e}^{k}=\left[y_{i}^{k}, y_{v}^{k}\right] ; \\
& i<v ; v \in L^{k} \cup U^{k} ; i \in U^{k} ; k \in K ; e=1, \cdots, n_{i} \\
& z_{i e}^{k} \in W_{i e}^{k}=\left[y_{u}^{k}, y_{i}^{k}\right] ; \\
& u<i ; u \in L^{k} \cup U^{k} ; i \in L^{k} ; k \in K ; e=1, \cdots, n_{i}
\end{aligned}
$$

\subsection{Heuristic Algorithm}

By applying the above procedure for converting buffer constraints, (9) (14), into time window constraints, the formulation becomes a scheduling problem with precedence and time window constraints. This section proposes a heuristic algorithm for solving this formulation. To precisely describe the heuristic algorithm, some notations and modifications to Nguyen and Kim (2009) are required as follows: in the initial stage, we suppose that $y_{i}^{k}$ variables equal to $s_{i}^{k}$ variables $k=1, \cdots,|K|, i$ $=1, \cdots, m_{k}$ are given. In addition, let $z_{i e}^{k} \mathrm{~s}$ be set to $L\left(W_{i}^{k}\right)$. Then, events are sequenced in an increasing order of $y_{i}^{k}$, and the sequence of events $\left(z_{i e}^{k} \mathrm{~s}\right)$ in $T$ is set the same as that of the $y_{i}^{k}$ variables.

Denote the $f^{\text {th }}$ event in sequence $T$ as event $(f)$, the event time of event ( $e$ ) as $z_{e}$ for the operation of vehicles, the time required for a vehicle to be ready for event $(f)$ after it experiences event $(e)$ as $c_{e f}$ (which corresponds to the notation of $c_{k i e}^{l i f}$ ), the pure travel time from the location of event (e) to the location of event $(f)$ as $t_{e f}$, the decision variable for the assignment of event $(e)$ to event (f) as $x_{e f}$, and the time window of $z_{e}$ as $W_{e}$.

Let $T_{\xi}$ be a subset of $T$ that includes only the first $\xi$ events in the sequence, and let $L_{\xi}$ and $U_{\xi}$ be subsets of $T_{\xi}$ that include only loading and unloading tasks, respectively. Then, the constraint subset $\xi$ of (2)-(4) and (16)(17) can be written as follows:

$$
\begin{aligned}
& \text { Constraint subset } \xi \\
& \sum_{e \in S \cup T_{\xi}} x_{e f}=1, \quad \forall f \in D \cup T_{\xi} \\
& \sum_{f \in D \cup T_{\xi}} x_{e f}=1, \forall e \in S \cup T_{\xi} \\
& z_{f}-\left(z_{e}+c_{e f}\right) \geq M\left(x_{e f}-1\right), \forall e \in S \cup T_{\xi} \\
& \qquad \forall e \in S \cup T_{\xi} \quad \text { and } f \in T_{\xi} \\
& z_{e} \in W_{e}, \forall e \in L_{\xi} \cup U_{\xi} \\
& x_{e f}=0 \text { or } 1, \forall e \in S \cup T_{\xi} \text { and } f \in D \cup T_{\xi}
\end{aligned}
$$

Then, the following algorithm assigns vehicles to
- Heuristic solution procedure for solving the scheduling problem with precedence and time window constraints.

Step 0: Initializing. Set $y_{i}^{k}=s_{i}^{k}, i=1, \cdots, m_{k}, k \in K$. Also, evaluate the time window for each $z_{i e}^{k}$. Set $z_{i e}^{k}=L\left(W_{i e}^{k}\right)$, and set $z_{i e}^{k U}=U\left(W_{i e}^{k}\right)$ for all $i \in$ $L^{k} \cup U^{k}, e=1, \cdots, n_{i}, k \in K$. Let $z_{0 i}^{O}=0$, for all $i \in V$. Start $\xi=0$.

Step 1: Next Task. $\xi=\xi+1$. If $\xi>m$ (the total number of tasks in sequence $T$ ), then go to Step 5 . Otherwise, sequence the events in the increasing order of $y_{i}^{k}$, and the sequence of events $\left(z_{i e}^{k} \mathrm{~s}\right)$ in $T$ is set the same as that of $y_{i}^{k}$ variables, and then go to Step 2.

Step 2: Feasibility Check.

Step 2.1: Check the existence of a feasible solution to constraints (18)-(20). If there is a feasible solution, then go to Step 2.2. Otherwise, go to Step 3.1 .

Step 2.2: Check whether the event time of event $(\xi)$ for vehicles satisfies constraint (21). If it is satisfied, then go to Step 4. Otherwise, go to Step 3.2.

Step 3: Delaying Event Time.

Step 3.1: Let the event time of event $(\xi)$ for vehicles be $z_{\lambda \mu}^{\gamma}\left(=z_{\xi}\right)$.

Let $\pi_{e^{*} \xi}=\min _{e \in S \cup T_{\xi-1}}\left[\max \left\{c_{e \xi}-\left(z_{\xi}-z_{e}\right), 0\right\}\right]$.

Then, $z_{\lambda \mu}^{\gamma}=z_{\lambda \mu}^{\gamma}+\pi_{e^{*} \xi}$. Go to Step 2.1.

Step 3.2: Let the event time of event $(\xi)$ for QC be $y_{\lambda}^{\gamma}$, and the event time of $z_{\xi}^{U}$ be $y_{\sigma}^{\gamma}$.

Let $\pi_{\xi}=z_{\xi}-z_{\xi}^{U}$. Then, $y_{r}^{\gamma}=y_{r}^{\gamma}+\pi_{\xi}$, for $r \geq$ $\sigma$ (Note $\sigma=\lambda$ for $\xi \in L_{\xi}$ ). Update $z_{r p}^{\gamma}=z_{r p}^{\gamma}+\pi_{\xi}$, for $r \geq \lambda, p=\mu+1, \cdots, n_{r}$ (Note $\left.z_{r p}^{\gamma} \neq 0\right)$, and $z_{r p}^{\gamma U}=z_{r p}^{\gamma U}+\pi_{\xi}$, for $r \geq \lambda, p=\mu, \cdots, n_{r}$. Go to Step 2.2.

Step 4: Time Window Adjustment.

Let $\underline{z}_{\lambda \mu}^{\gamma}$ be an event time of $z_{\lambda \mu}^{\gamma}$ in the previous stage.

If $\xi \in L_{\xi}$ and the upper bound of a related preceding unloading task $r, p=1, \cdots, n_{r}, z_{r p}^{\gamma U} \in\left[\underline{z}_{\lambda \mu}^{\gamma}, z_{\lambda \mu}^{\gamma}\right)$, then it must be extended to $z_{r p}^{\gamma U}=z_{\lambda \mu}^{\gamma}$. Go to Step 1 .

If $\xi \mathrm{C} U_{\xi}$ and the lower bound of a related succeeding loading task $r, p=1, \cdots, n_{r}, z_{r p}^{\gamma} \in\left(z_{\lambda \mu}^{\gamma}, z_{\lambda \mu}^{\gamma U}\right]$, then it must be extended to $z_{r p}^{\gamma}=z_{\lambda \mu}^{\gamma}$. Go to Step 1 .

Step 5: Task Assignment. Evaluate $t_{e f}$. Solve the assignment problem with the objective of minimizing the total travel distance subject to constraint subset $|T|$. Stop. tasks. 
Feasibility Check: This step consists of two steps. In the first step, for given values of $z_{i e}^{k}$ variables, the feasibility can be checked by solving a maximum cardinality matching problem in the bipartite graph (Evans and Minieka 1992). When the maximum cardinality is the same as $\{S \cup T\}$, it can be concluded that the constraint subset $\xi$ of (18)-(20) has a feasible solution. Otherwise, it is infeasible. For a given feasible solution in Step 2.1, Step 2.2 checks whether $z_{i e}^{k}$ does not exceed the upper bound of the time window.

Delaying Event Time: To satisfy the constraint subset of (18)-(20), one or more $x_{\text {kie }}^{\text {lif }}$ must be allowed to become 1 by relaxing constraint (20). That is, the event time of event $(\xi)$ is delayed by such an amount that at least one $x_{e \xi}$, for $e<\xi$, becomes 1 , which is denoted as $\pi_{e^{*} \xi}$. Further, in some cases, for satisfying the constraint subsets of (18)-(20), it may be necessary to increase $z_{i e}^{k}$ beyond the upper bound of the time window (constraint subset (21)), in which the event time for QCs, $y_{r}^{\gamma}$, must be increased, and the time windows must be updated simultaneously. In the process, when the current constraint subset becomes feasible, the iteration is stopped, and we proceed to the next stage.

Time Window Adjustment: After obtaining a feasible solution for constraint subset $\xi$ of (18)-(22), the time window must be adjusted if possible. When a feasible solution is found, the event time $z_{\lambda \mu}^{\gamma}$ of event $(\xi)$ will be determined. Then, the time window of the event related to that of event $(\xi)$ may be adjusted by extending its bound. For a loading event $(\xi)$, the upper bound of the related preceding unloading tasks may be extended. For an unloading event $(\xi)$, the lower bound of the re lated succeeding loading tasks may be extended.

An application of the heuristic algorithm to the example in Table 1, which includes QC $1, b_{1}=5, m_{1}=4$, and two vehicles, is illustrated as follows. The event numbers, $e$ and $f$, in Table 3 correspond to task numbers for QC 1.

Table 3. The time required for a vehicle to be ready for event (f) after it experiences event (e), cef, (in seconds).

\begin{tabular}{|ccccccccccccc|}
\hline To $f$ & $0^{*}$ & 1 & 2 & 3 & 4 & 5 & 6 & 7 & 8 & 9 & 10 \\
Frome & \multicolumn{1}{c}{$\left(a_{11}^{1}\right)\left(a_{12}^{1}\right)\left(a_{13}^{1}\right)\left(a_{21}^{1}\right)\left(a_{22}^{1}\right)\left(a_{31}^{1}\right)\left(a_{32}^{1}\right)\left(a_{41}^{1}\right)\left(a_{42}^{1}\right)\left(a_{43}^{1}\right)$} \\
\hline \hline 0 & - & 180 & 200 & 180 & 80 & 80 & 200 & 180 & 80 & 80 & 80 \\
1 & - & - & 220 & 200 & 0 & 0 & 240 & 220 & 0 & 0 & 0 \\
2 & - & - & - & 240 & 0 & 0 & 240 & 220 & 0 & 0 & 0 \\
3 & - & - & - & - & 0 & 0 & 240 & 220 & 0 & 0 & 0 \\
4 & - & - & - & - & - & 220 & 260 & 240 & 220 & 220 & 220 \\
5 & - & - & - & - & - & - & 240 & 260 & 240 & 240 & 240 \\
6 & - & - & - & - & - & - & - & 220 & 0 & 0 & 0 \\
7 & - & - & - & - & - & - & - & - & 0 & 0 & 0 \\
8 & - & - & - & - & - & - & - & - & - & 220 & 220 \\
9 & - & - & - & - & - & - & - & - & - & - & 240 \\
\hline
\end{tabular}

Note: ${ }^{*}$ Initial starting event of vehicles.
Step 0: Initializing. Set $y_{i}^{1}=(0,230,230,460)$. The time windows for the loading operations are $z_{1 e}^{1}=0$, $e=1, \cdots, 3$; and $0 \leq z_{3 e}^{1} \leq 230, e=1,2$; while the time windows for the unloading operations are $230 \leq z_{2 e}^{1} \leq \infty, e=1,2$; and $460 \leq z_{4 e}^{1} \leq \infty, e$ $=1, \cdots, 3$. Thus, $z_{i e}^{1}=(0,0,0,230,230,0,0$, $460,460,460)$, and $z_{i e}^{1 U}=(0,0,0, \infty, \infty, 230$, $230, \infty, \infty, \infty), i=1, \cdots, 4, e=1, \cdots, n_{i}$. Let $z_{0 i}^{o}=0, i=1,2$. Start $\xi=0$.

Step 1: Next Task. $\boldsymbol{\xi}=\boldsymbol{\xi}+1=1$. Sequence the events in the increasing order of $y_{i}^{k}, y_{i}^{1}=\left(y_{1}^{1}, y_{2}^{1}, y_{3}^{1}, y_{4}^{1}\right)$, and the sequence of events $\left(z_{i e}^{k} \mathrm{~s}\right)$ in $T$ is $z_{i e}^{1}=$ $\left(z_{11}^{1}, z_{12}^{1}, z_{13}^{1}, z_{21}^{1}, z_{22}^{1}, z_{31}^{1}, z_{32}^{1}, z_{41}^{1}, z_{42}^{1}, z_{43}^{1}\right)$.

\section{Step 2: Feasibility Check.}

Step 2.1: There is no feasible solution to constraints (18)-(20).

Step 3: Delaying Event Time.

Step 3.1: Calculate $\pi_{e^{*} 1}=\min \{180-(0-0) ; 180-$ $(0-0)\}=180 \stackrel{e}{=} \pi_{1^{*} 1}$. Thus, $z_{11}^{1}=180$.

Step 2: Feasibility Check.

Step 2.1: At least, a feasible solution to constraints (18) (20) can be found.

Step 2.2: Compare $z_{11}^{1}$ with its upper bound. It does not satisfy constraint (21).

Step 3: Delaying Event Time.

Step 3.2. Let $\pi_{1}=180-0=180$. Then, $y_{i}^{1}=(180$, $410,410,640)$, and update $z_{i e}^{1}=(180,0,0,410$, $410,0,0,640,640,640)$, and $z_{i e}^{1 U}=(180,180$, $180, \infty, \infty, 410,410, \infty, \infty, \infty)$.

Step 2: Feasibility Check.

Step 2.2. Compare $z_{11}^{1}$ with its upper bound. It satisfies constraint (21).

Step 4: Time window Adjustment. There is no preceding unloading task of task $(\xi)$. Thus, there is no adjustment of time windows.

Step 1: Next Task. $\xi=2$. Sequence the events in the increasing order of $y_{i}^{k}, y_{i}^{1}=\left(y_{1}^{1}, y_{2}^{1}, y_{3}^{1}, y_{4}^{1}\right)$, and the sequence of events $\left(z_{i e}^{k} \mathrm{~s}\right)$ in $T$ is $z_{i e}^{1}=\left(z_{12}^{1}, z_{13}^{1}\right.$, $\left.z_{11}^{1}, z_{21}^{1}, z_{22}^{1}, z_{31}^{1}, z_{32}^{1}, z_{41}^{1}, z_{42}^{1}, z_{43}^{1}\right)$.

Step 2: Feasibility Check.

Step 2.1: There is no feasible solution to constraints (18)-(20). 
Step 3: Delaying Event Time.

Step 3.1. Calculate $\pi_{*^{*}}=\min \{220-(0-180) ; 200-$ $(0-0)\}=200=\pi_{2^{*} 2}$. Thus, $z_{12}^{1}=200$.

Step 2: Feasibility Check.

Step 2.1: At least, a feasible solution to constraints (18)-(20) can be found.

Step 2.2: Compare $z_{12}^{1}$ with its upper bound. It does not satisfy constraint (21).

Step 3: Delaying Event Time.

Step 3.2: Let $\pi_{2}=200-180=20$. Then, $y_{i}^{1}=(200$, $430,430,660)$, and update $z_{i e}^{1}=(180$, 200, 0, 430, 430, 0, 0, 660, 660, 660), and $z_{i e}^{1 U}=(180,200,200, \infty, \infty, 430$, $430, \infty, \infty, \infty)$.

Step 2: Feasibility Check.

Step 2.2: Compare $z_{12}^{1}$ with its upper bound. It satisfies constraint (21).

Step 4: Time window Adjustment. There is no preceding unloading task of task $(\xi)$. Thus, there is no adjustment of time windows.

Step 1: Next Task. $\boldsymbol{\xi}=3$. Sequence the events in the increasing order of $y_{i}^{k}, y_{i}^{1}=\left(y_{1}^{1}, y_{2}^{1}, y_{3}^{1}, y_{4}^{1}\right)$, and the sequence of events $\left(z_{i e}^{k} \mathrm{~s}\right)$ in $T$ is $z_{i e}^{1}=\left(z_{13}^{1}, z_{11}^{1}\right.$, $\left.z_{12}^{1}, z_{21}^{1}, z_{22}^{1}, z_{31}^{1}, z_{32}^{1}, z_{41}^{1}, z_{42}^{1}, z_{43}^{1}\right)$.

Step 2: Feasibility Check.

Step 2.1: There is no feasible solution to constraints (18)-(20).

Step 3: Delaying Event Time.

Step 3.1: Calculate $\pi_{e^{*} 3}=\min \{200-(0-180) ; 240-$ $(0-200)\}=380=\pi_{1^{*} 3}$. Thus, $z_{13}^{1}=380$.

Step 2: Feasibility Check.

Step 2.1: At least, a feasible solution to constraints (18)-(20) can be found.

Step 2.2: Compare $z_{13}^{1}$ with its upper bound. It does not satisfy constraint (21).

Step 3: Delaying Event Time.

Step 3.2: Let $\pi_{3}=380-200=180$. Then, $y_{i}^{1}=$ $(380,610,610,840)$, and update $z_{i e}^{1}=$ $(180,200,380,610,610,0,0,840$, $840,840)$, and $z_{i e}^{1 U}=(180,200,380, \infty$, $\infty, 610,610, \infty, \infty, \infty)$.

Step 2: Feasibility Check.

Step 2.2: Compare $z_{13}^{1}$ with its upper bound. It satisfies constraint (21).

Step 4: Time window Adjustment. There is no preceding unloading task of task $(\xi)$. Thus, there is no ad- justment of time windows.

Step 1: Next Task. $\xi=4$. Sequence the events in the increasing order of $y_{i}^{k}, y_{i}^{1}=\left(y_{1}^{1}, y_{2}^{1}, y_{3}^{1}, y_{4}^{1}\right)$, and the sequence of events $\left(z_{i e}^{k} \mathrm{~s}\right)$ in $T$ is $z_{i e}^{1}=\left(z_{11}^{1}\right.$, $\left.z_{12}^{1}, z_{13}^{1}, z_{21}^{1}, z_{22}^{1}, z_{31}^{1}, z_{32}^{1}, z_{41}^{1}, z_{42}^{1}, z_{43}^{1}\right)$.

Step 2: Feasibility Check.

Step 2.1: At least, a feasible solution to constraints (18)-(20) can be found.

Step 2.2: Compare $z_{21}^{1}$ with its upper bound. It satisfies constraint (21).

Step 4: Time window Adjustment. There is a succeeding loading task of task $(\xi)$. However, since its lower bound $z_{31}^{1}$ is less than $z_{21}^{1}$, it cannot be extended.

Step 1: Next Task. $\xi=5$. Sequence the events in the increasing order of $y_{i}^{k}, y_{i}^{1}=\left(y_{1}^{1}, y_{2}^{1}, y_{3}^{1}, y_{4}^{1}\right)$, and the sequence of events $\left(z_{i e}^{k} \mathrm{~s}\right)$ in $T$ is $z_{i e}^{1}=\left(z_{11}^{1}\right.$, $\left.z_{12}^{1}, z_{13}^{1}, z_{22}^{1}, z_{21}^{1}, z_{31}^{1}, z_{32}^{1}, z_{41}^{1}, z_{42}^{1}, z_{43}^{1}\right)$.

Step 2: Feasibility Check.

Step 2.1: At least, a feasible solution to constraints (18)-(20) can be found.

Step 2.2: Compare $z_{22}^{1}$ with its upper bound. It satisfies constraint (21)

Step 4: Time window Adjustment. There is a succeeding loading task of task $(\xi)$. However, since its lower bound $z_{31}^{1}$ is less than $z_{22}^{1}$, it cannot be extended.

This process is repeated until $\xi$ is greater than the total number of containers (10). Finally, the solution obtained by the assignment problem is as follows: $x_{001}^{111}=$ $x_{111}^{113}=x_{113}^{121}=x_{121}^{132}=x_{132}^{141}=x_{141}^{143}=x_{143}^{F 01}=x_{002}^{112}=x_{112}^{122}=x_{122}^{131}=x_{131}^{142}=$ $x_{142}^{F 02}=1$, and all the other, $x_{\text {kie }}^{l i f}=0$; and $y_{i}^{1}=(380,610$, $850,1080), z_{i e}^{1}=(180,200,380,610,610,850,850,1080$, 1080,1300 ) for $i=1, \cdots, 4 ; e=1, \cdots, n_{i}$.

Figure 6 illustrates the solution procedure. The final solution can be explained as follows: from the initial location, vehicle 1 and vehicle 2 start to travel empty to the pickup positions for the $1^{\text {st }}$ and $2^{\text {nd }}$ containers of the $1^{\text {st }}$ loading task, respectively, and perform the loaded travel for the tasks. After vehicle 1 moves the $1^{\text {st }}$ container, it goes to move the $3^{\text {rd }}$ container of the $1^{\text {st }}$ loading task. Because the $1^{\text {st }}$ loading task of QC 1 consists of three containers that are transferred by the triple lift, QC 1 must wait $380 \mathrm{~s}$ for the arrival of the containers at the buffer. Next, vehicle 1 waits $230 \mathrm{~s}$ for picking up the $1^{\text {st }}$ container of the $2^{\text {nd }}$ unloading task and delivers it to storage block. Then, vehicle 1 goes to the storage block of the $2^{\text {nd }}$ container of the $3^{\text {rd }}$ loading task to transfer the container to QC 1 . After then, it waits $230 \mathrm{~s}$ for the arrival of the $1^{\text {st }}$ container of the $4^{\text {th }}$ unloading task, and goes to the storage block of its container. Finally, it moves to QC 1 for delivering the $3^{\text {rd }}$ container of the $4^{\text {th }}$ unloading task to its storage block. In a similar way, after 


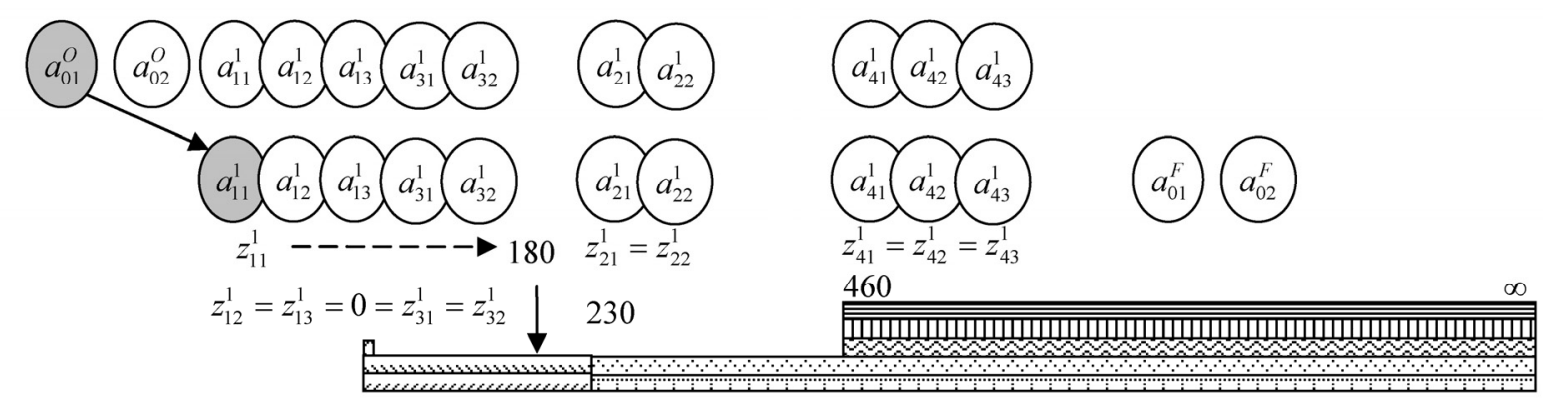

(a) Assignment of the $1^{\text {st }}$ container of the $1^{\text {st }}$ task $(\xi=1)$

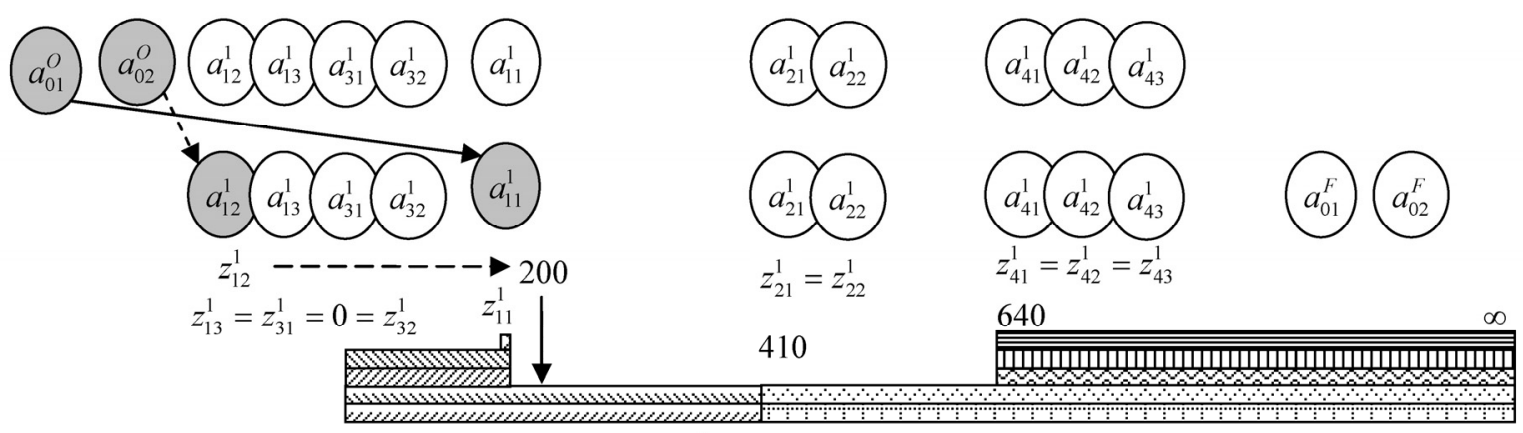

(b) Assignment of the $2^{\text {nd }}$ container of the $1^{\text {st }}$ task $(\xi=2)$

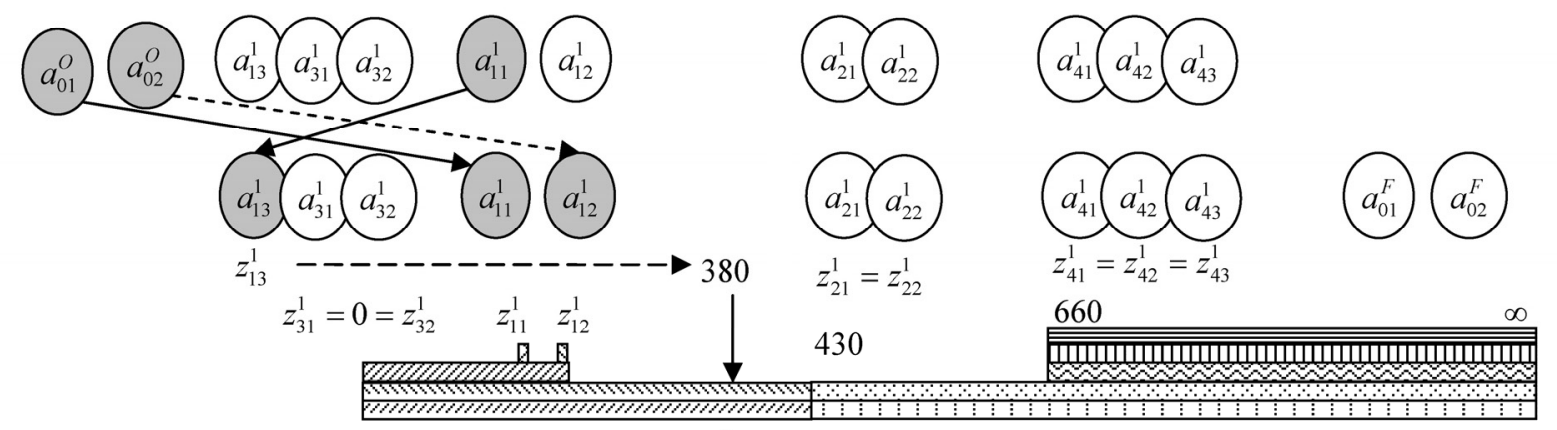

(c) Assignment of the $3^{\text {rd }}$ container of the $1^{\text {st }}$ task $(\xi=3)$

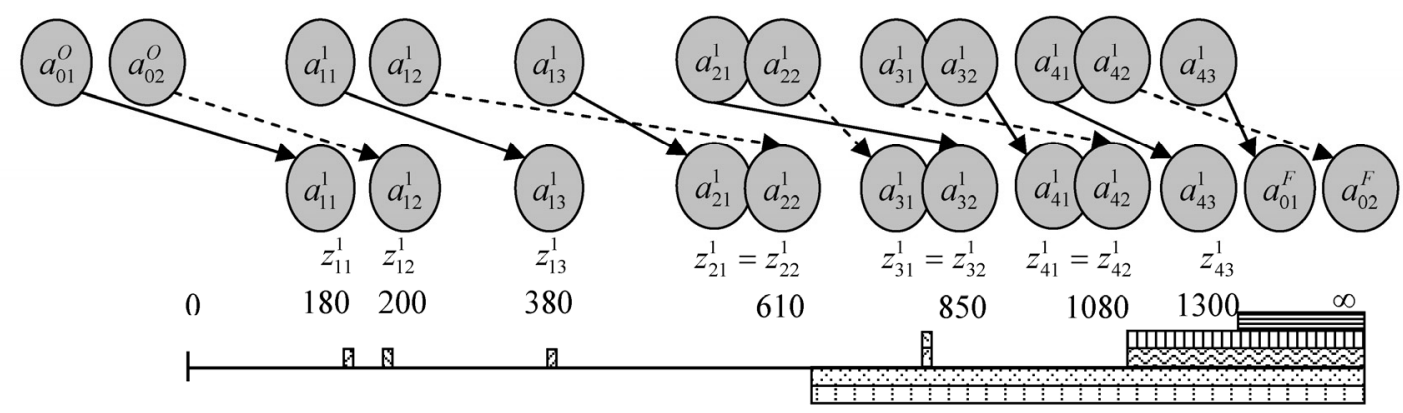

(d) Assignment of the containers of all tasks $(\xi=10)$
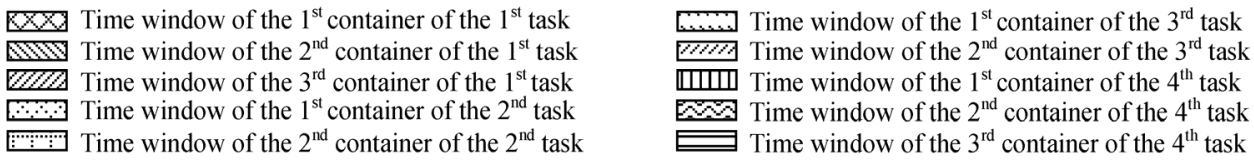

Figure 6. An illustration of the heuristic algorithm. 
Table 4. Comparison between results by the heuristic algorithm and those by formulations (1)-(15).

\begin{tabular}{|c|c|c|c|c|c|c|c|}
\hline \multirow[b]{2}{*}{ No. } & \multirow[b]{2}{*}{ Problem } & \multicolumn{3}{|c|}{ Objective Value } & \multicolumn{3}{|c|}{ Computational Time (s) } \\
\hline & & $\begin{array}{c}\text { Heuristic } \\
\text { algorithm (A) }\end{array}$ & $\begin{array}{c}\text { Optimal } \\
\text { solution (B) }\end{array}$ & $\begin{array}{c}\text { Ratio } \\
(=\mathrm{A} / \mathrm{B})\end{array}$ & $\begin{array}{c}\text { Heuristic } \\
\text { algorithm (C) }\end{array}$ & $\begin{array}{c}\text { Optimal } \\
\text { solution (D) }\end{array}$ & $\begin{array}{c}\text { Ratio } \\
\text { (= C/D) }\end{array}$ \\
\hline 1 & Triple(3-1-4-3) ${ }^{*}$ & 3200 & 2540 & 1.260 & 0.39 & 60 & 0.0065 \\
\hline 2 & Triple(4-1-4-3) & 2880 & 2280 & 1.263 & 0.578 & 58 & 0.0100 \\
\hline 3 & Triple(5-1-4-3) & 1820 & 1820 & 1.000 & 0.391 & 53 & 0.0074 \\
\hline 4 & Triple(6-1-4-3) & 1760 & 1760 & 1.000 & 0.265 & 21 & 0.0126 \\
\hline 5 & Triple(3-1-4-6) & 3100 & 2540 & 1.220 & 0.453 & 372 & 0.0012 \\
\hline 6 & Triple(4-1-4-6) & 2680 & 2280 & 1.175 & 0.469 & 95 & 0.0049 \\
\hline 7 & Triple(5-1-4-6) & 1820 & 1820 & 1.000 & 0.407 & 58 & 0.0070 \\
\hline 8 & Triple(6-1-4-6) & 1760 & 1760 & 1.000 & 0.406 & 21 & 0.0193 \\
\hline 9 & Triple(5-1-6-3) & 3420 & 2740 & 1.248 & 0.547 & 1037 & 0.0005 \\
\hline 10 & Triple(6-1-6-3) & 2660 & 2480 & 1.073 & 0.328 & 63 & 0.0052 \\
\hline 11 & Triple(7-1-6-3) & 2400 & 2400 & 1.000 & 0.297 & 84 & 0.0035 \\
\hline 12 & Triple(8-1-6-3) & 2320 & 2320 & 1.000 & 0.36 & 68 & 0.0053 \\
\hline 13 & Triple(9-1-6-3) & 2240 & 2240 & 1.000 & 0.359 & 90 & 0.0040 \\
\hline 14 & Triple(5-1-6-6) & 2700 & 2700 & 1.000 & 0.64 & 162 & 0.0040 \\
\hline 15 & Triple(6-1-6-6) & 2660 & 2480 & 1.073 & 0.578 & 101 & 0.0057 \\
\hline 16 & Triple(7-1-6-6) & 2400 & 2400 & 1.000 & 0.531 & 53 & 0.0100 \\
\hline 17 & Triple(8-1-6-6) & 2320 & 2320 & 1.000 & 0.516 & 98 & 0.0053 \\
\hline 18 & Triple(9-1-6-6) & 2240 & 2240 & 1.000 & 0.468 & 36 & 0.0130 \\
\hline 19 & Triple(6-1-8-3) & 3560 & 3200 & 1.113 & 0.797 & 282 & 0.0028 \\
\hline 20 & Triple(7-1-8-3) & 3120 & 3120 & 1.000 & 0.469 & 88 & 0.0053 \\
\hline 21 & Triple(8-1-8-3) & 3040 & 3040 & 1.000 & 0.594 & 258 & 0.0023 \\
\hline 22 & Triple(9-1-8-3) & 2960 & 2960 & 1.000 & 0.453 & 125 & 0.0036 \\
\hline 23 & Triple(6-1-8-6) & 3560 & 3200 & 1.113 & 0.844 & 210 & 0.0040 \\
\hline 24 & Triple(7-1-8-6) & 3120 & 3120 & 1.000 & 0.578 & 352 & 0.0016 \\
\hline 25 & Triple(8-1-8-6) & 3040 & 3040 & 1.000 & 0.656 & 229 & 0.0029 \\
\hline 26 & Triple(9-1-8-6) & 2960 & 2960 & 1.000 & 0.562 & 111 & 0.0051 \\
\hline 27 & Triple(7-1-10-3) & 3840 & 3840 & 1.000 & 0.531 & 677 & 0.0008 \\
\hline 28 & Triple(8-1-10-3) & 3760 & 3760 & 1.000 & 0.531 & 421 & 0.0013 \\
\hline 29 & Triple(9-1-10-3) & 3680 & 3680 & 1.000 & 0.484 & 437 & 0.0011 \\
\hline 30 & Triple(10-1-10-3) & 3600 & 3600 & 1.000 & 0.672 & 464 & 0.0014 \\
\hline 31 & Triple(11-1-10-3) & 3520 & 3520 & 1.000 & 0.485 & 463 & 0.0010 \\
\hline 32 & Triple(12-1-10-3) & 3440 & 3440 & 1.000 & 0.484 & 247 & 0.0020 \\
\hline 33 & Triple(7-1-10-6) & 3840 & 3840 & 1.000 & 0.703 & 8997 & 0.0001 \\
\hline 34 & Triple(8-1-10-6) & 3760 & 3760 & 1.000 & 0.563 & 479 & 0.0012 \\
\hline 35 & Triple(9-1-10-6) & 3680 & 3680 & 1.000 & 0.547 & 296 & 0.0018 \\
\hline 36 & Triple(10-1-10-6) & 3600 & 3600 & 1.000 & 0.609 & 466 & 0.0013 \\
\hline 37 & Triple(11-1-10-6) & 3520 & 3520 & 1.000 & 0.656 & 348 & 0.0019 \\
\hline 38 & Triple(12-1-10-6) & 3440 & 3440 & 1.000 & 0.625 & 200 & 0.0031 \\
\hline \multicolumn{4}{|c|}{ Average } & 1.040 & & & 0.0045 \\
\hline \multicolumn{4}{|c|}{ Min } & 1.000 & & & 0.0001 \\
\hline \multicolumn{4}{|c|}{ Max } & 1.263 & & & 0.0193 \\
\hline
\end{tabular}

Note: ${ }^{*}$ Number of vehicles per QC-Number of QCs-Number of operations per each QC-Buffer capacity. 
vehicle 2 moves the $2^{\text {nd }}$ container of the $1^{\text {st }}$ loading task, it waits $410 \mathrm{~s}$ for the arrival of the $2^{\text {nd }}$ container of the $2^{\text {nd }}$ unloading task and delivers it to the storage block. Next, it goes to the storage block of the $1^{\text {st }}$ container of the $3^{\text {rd }}$ loading task and transfers the container to QC 1 . Then, vehicle 2 waits $230 \mathrm{~s}$ for the arrival of the $2^{\text {nd }}$ container of the $4^{\text {th }}$ unloading task, and finally goes to the storage block of its container.

\section{NUMERICAL EXPERIMENTS}

This section discusses a numerical experiment to compare the performance of the heuristic algorithm with that of the optimizing method and analyzes the properties of the heuristic algorithm proposed in this study.

\subsection{Comparing the Heuristic Algorithm and the Opt- imizing Method}

A number of problems were generated to compare the optimal solutions obtained from the formulations (1) (15) with those from the heuristic algorithm. In the generated problems, the total number of vehicles, the number of operations for each QC, and the buffer capacity ranged from 3 to 12 , from 4 to 10 , and from 3 to 6 , respectively. Moreover, the number of containers per operation was three to represent triple lifts. The formulations (1)-(15) were solved by ILOG CPLEX ${ }^{\circledR}$ 10.0.0. The results of the generated problems are shown in Table 4 . The ratios of the objective values obtained by the heuristic algorithm to those obtained by ILOG CPLEX ${ }^{\circledR}$ ranged from 1.000 to 1.263 , with an average value of 1.040. The ratios of the computational times for the heuristic algorithm to those of ILOG CPLEX ${ }^{\circledR}$ ranged from 0.0001 to 0.0193 , with an average value of 0.0045 .

\subsection{Sensitivity Analysis}

In order to analyze and evaluate the effects of the number of vehicles and the buffer capacity on the sum of the QC delay times, total travel time for the vehicles, and completion time, various numerical experiments were conducted as follows: the number of QCs, the number of operations per QC, and the number of containers for each operation were 2, 20, and 3, respectively. The number of vehicles was varied from 8 to 14 . The buffer capacities under the QCs in the apron were considered in the case of no buffer (buffer capacity of zero) and in the cases of buffers from 3 to 6 . The performances of the experiments were analyzed in terms of the sum of the QC delay times, total travel time of the vehicles, and completion time. For each combination, ten problems were generated randomly. Therefore, the total number of problems solved was 350 . The results of these numerical experiments are shown in Figure 11-15.

In addition, this study compared the QC productivity, the sum of the QC delay times, total travel time of the vehicles, and the average time loss caused by the lateness of arrival of vehicles for each operation between different types of operations by QCs (single, tandem, and triple lift). For this purpose, it was assumed that there was no buffer space under the QCs in the apron. The results of this comparison are shown in Figure 7-10.

Figure 7 illustrates the comparison between different types of QC operations (single, tandem, and triple lift) of the QC productivity. It was shown that the QC productivity of tandem lift was best as the number of vehicles was small. While, the QC productivity of triple lift was best as the number of vehicles was large. When the number of vehicles increased in this study, the QC productivity of single lift did not change and that of tandem lift increased slightly. However, the QC productivity of triple lift increased rapidly as the number of vehicles increased. When the number of vehicles (12 as shown in Figure 7) was sufficient to support tandem and triple lifts by QCs, the ratios of the QC productivity of tandem and triple lift to that of single lift were mostly twice and three times, respectively.

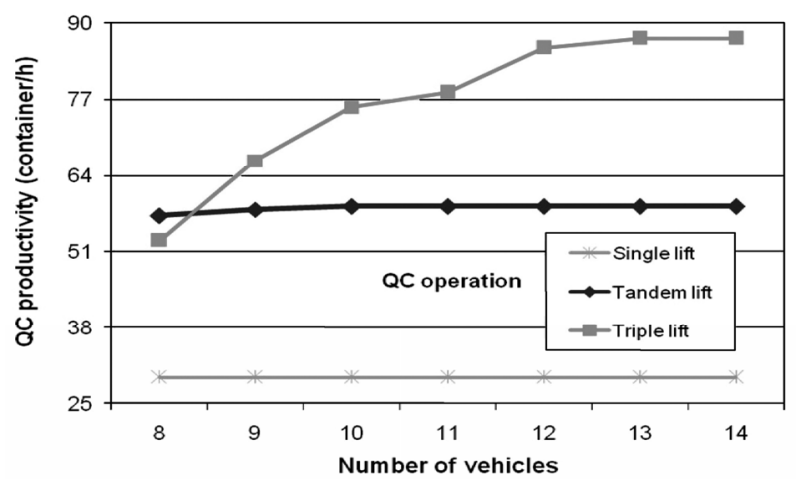

Figure 7. Comparison of QC productivity among various of QC operations.

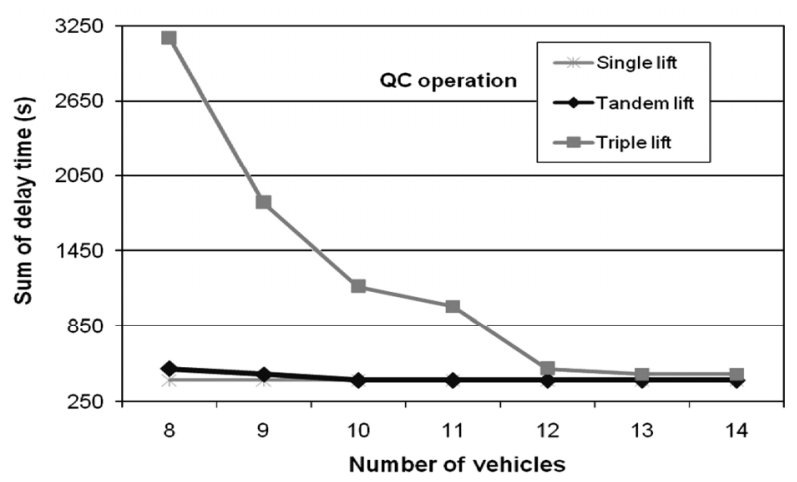

Figure 8. Comparison of the sum of the $\mathrm{QC}$ delay times among various types of $\mathrm{QC}$ operations.

The comparison between different types of QC operations of the sum of the QC delay times is presented in Figure 8. The sum of the QC delay times of single lift 
was best because the number of containers handled by single lifts was a half and one third in comparison with those by tandem and triple lifts. When the number of vehicles increased, the sum of the QC delay times of tandem lift reduced slightly, while the sum of the QC delay times of triple lift reduced rapidly. When the Number of vehicles reached 12 (as shown in Figure 8), the reductions were negligible. Similarly, the comparisons of total travel time of vehicles are shown in Figure 9. Total travel time of vehicles of single lift by QCs was best. However, total travel time of vehicles of single, tandem, and triple lift by QCs did not change as the number of vehicles increased.

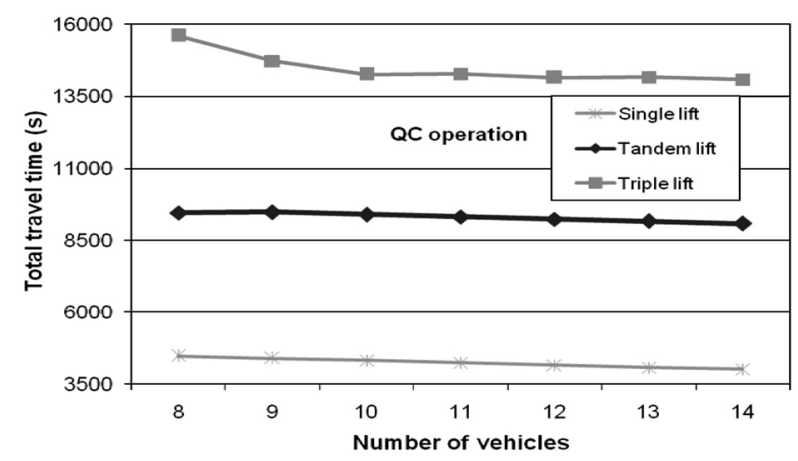

Figure 9. Comparison of total travel time of Vehicles among various types of QC operations.

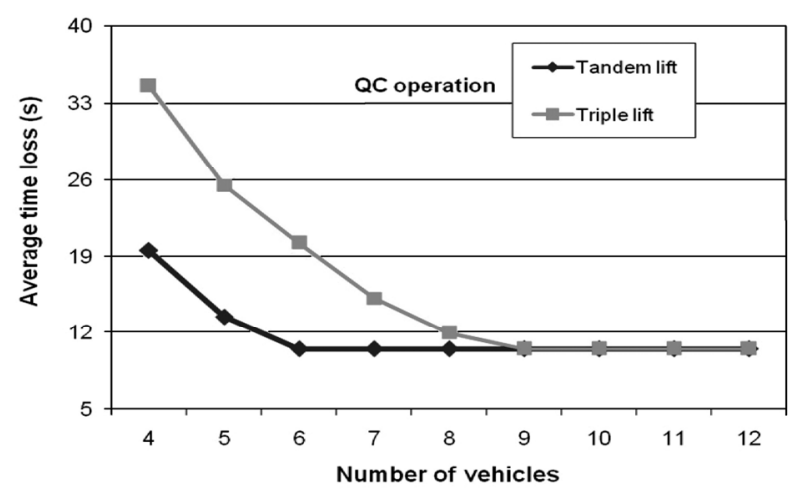

Figure 10. Comparison of the average time loss among various types of QC operations.

For the time loss caused by the lateness of arrival of vehicles, Figure 10 shows comparisons between two different types of QC operations for the average time loss. It was shown that the average time loss for tandem lifts by QCs was better than that for triple lifts by QCs. For example, when the number of vehicles was four, the average time loss for the tandem and the triple lifts of QCs were 19.50 (s) and 34.55 (s), respectively. However, the average time loss for both cases decreased rapidly with an increase in the number of vehicles. When the number of vehicles reached a value of nine (as shown in
Figure 10), the reductions were negligible, indicating that the number of vehicles was sufficient to support tandem and triple lifts by QCs.

The results from Figure 11 show the changes in the sum of the QC delay times for various buffer capacity values and different numbers of vehicles. For each buffer capacity value, the sum of the QC delay times decreased rapidly with an increase in the number of vehicles. When the number of vehicles exceeded a certain value (12 vehicles in Figure 11), the reductions were negligible, which was supported by a statistical test with a significance level of $5 \%$. This implies that the number of vehicles (12) was sufficient to support the triple lifts of the QCs. For changes in the buffer capacity, the sum of the QC delay times was reduced by increasing the buffer capacity. The case with no buffer was the worst. However, when the buffer capacity and the number of vehicles reached 5 and 12, respectively, the sum of the QC delay times was not affected significantly. Similarly, the changes in the completion time are shown in Figure 12. When the buffer capacity was larger than three, the completion time was slightly reduced with an increase in the number of vehicles. When the buffer capacity reached six, the completion time showed a considerable reduction in comparison with the others.

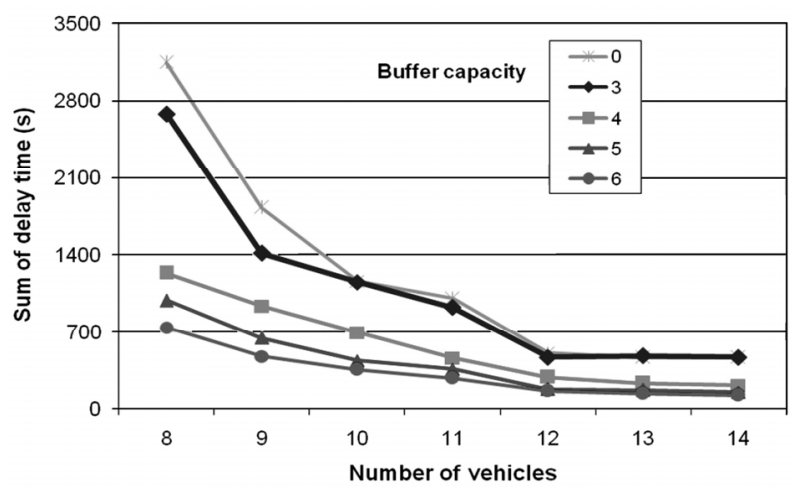

Figure 11. Effects of the number of vehicles on the sum of the QC delay times.

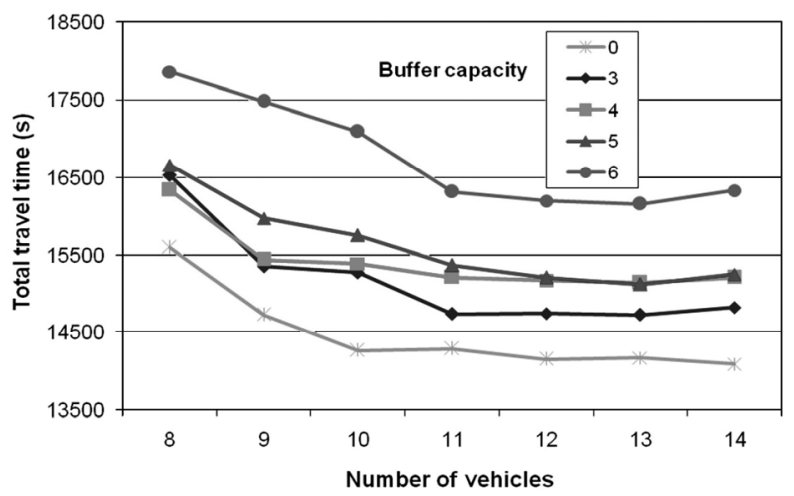

Figure 12. Effects of the number of vehicles on the Completion time. 


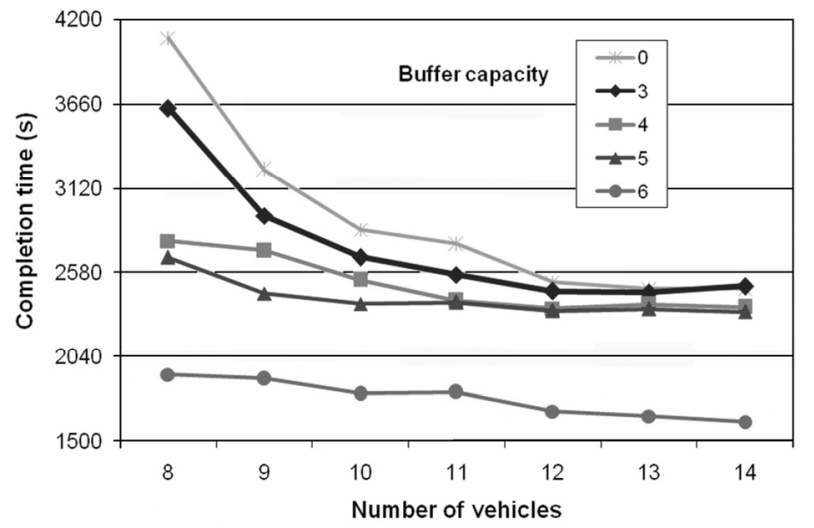

Figure 13. Effects of the number of vehicles on total travel time of vehicles.

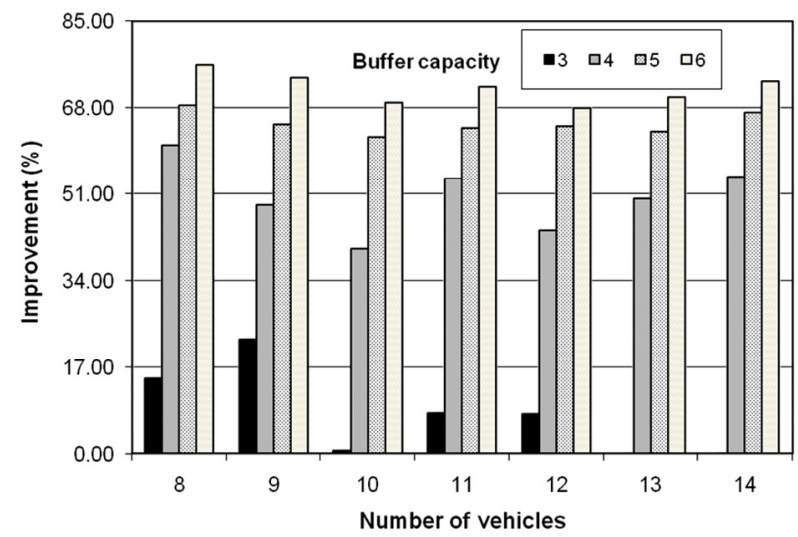

Figure 14. Improvements in the sum of the delay times for cases with 3 to 6 buffers compared with that with no buffer.

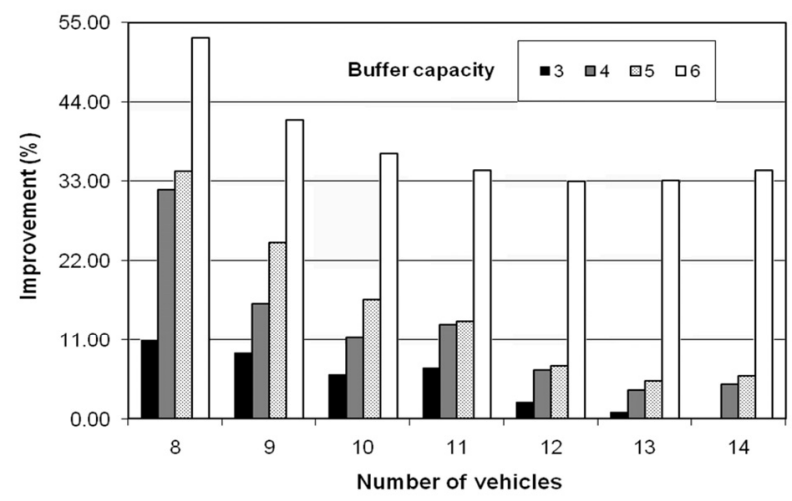

Figure 15. Improvements in the completion time for cases with 3 to 6 buffers compared with that with no buffer.

Figure 13 shows a comparison of the total travel times of the vehicles. Similarly, the total travel time of the vehicles decreased rapidly with an increase in the number of vehicles. However, the cases with smaller buffer sizes showed shorter travel distances than cases with larger buffer sizes. It can be concluded that the reduction in the objective of the delay time of the QCs was obtained at the cost of the other objective of the travel distance of the vehicles.

Figures 14 and 15 illustrate the differences in the sum of the QC delay times and completion times between the cases with buffer capacities from 3 to 6 and the case with no buffer. These differences increased with an increase in the buffer capacity. For example, when the number of vehicles was 12 , the differences in the delay times and completion times ranged from $7.8 \%$ to $68.0 \%$ and from $2.3 \%$ to $33.0 \%$, respectively, as the buffer capacity changed from 3 to 6 . This can be explained as an improvement between an ALV system with buffers and an AGV system with no buffer.

\section{CONCLUSIONS AND FURTHER STUDIES}

This study discussed a method for dispatching vehicles to support efficient multi-lifts by QCs, especially tandem and triple lifts. The problem was introduced as a scheduling problem with precedence and buffer constraints. A mixed integer programming model was proposed for the vehicle scheduling problem considering the multi-lifts of QCs. Then, a procedure for converting buffer constraints into time window constraints and a heuristic algorithm for solving the scheduling problem with precedence constraints and time window constraints were suggested. A number of numerical experiments were conducted to compare and analyze the proposed heuristic algorithm. First, several problems were generated to compare the optimal solution from the mathematical formulations with those from the heuristic algorithm. The results of this experiment showed that the ratio of the objective values obtained from the heuristic algorithm to the optimal objective values obtained from the ILOG CPLEX® was between 1.000 and 1.263 , with an average value of 1.040 . The ratio of the computational time for the heuristic algorithm to that of ILOG CPL EX ${ }^{\circledR}$ ranged from 0.0001 to 0.0193 , with an average value of 0.0045 . Therefore, it could be concluded that the heuristic algorithm showed good performance.

From the comparisons between different types of QC operations (single, tandem, and triple lift), it can be said that the QC productivity of tandem lift was best as the number of vehicles was small. While, the QC productivity of triple lift was best as the number of vehicles was large. For the time loss caused by the lateness of arrival of vehicles, the average time loss for tandem lifts by QCs was better than that for triple lifts by QCs. The average time loss for both cases decreased with an increase in the number of vehicles.

According to the sensitive analyses, both the sum of the QC delay times and the completion time decreased with an increase in the number of vehicles and the buffer capacity. However, the effect of the buffer size was significant when the number of vehicles was 
small, while the effect decreased with an increase in the number of vehicles. The performance of the ALV system with buffers was better than that of the AGV system with no buffer. For the total travel time of the vehicles, when the number of vehicles increased, the total travel time of the vehicles reduced. However, the cases with smaller buffer sizes showed shorter travel distances than cases with larger buffer sizes.

This study only evaluated and analyzed the heuristic algorithm as a dispatching method for vehicles in a static environment. Thus, a simulation study will be necessary to evaluate the performance of the algorithm proposed in this study in a dynamic environment. Moreover, the integrated scheduling problem for vehicle travel, YC operations, and QC operations may be addressed as a further extension of this study.

\section{ACKNOWLEDGMENTS}

This study was supported by the Korean Ministry of Education and Human Resources Development through the Regional Research Centers Program (Research Center for Logistics Information Technology).

\section{REFERENCES}

Bilge, U. and Ulusoy G. (1995), A time window approach to simultaneous scheduling of machines and material handling system in an FMS, Operations Research, 43, 1058-1070.

Bish, E. K., Chen F. Y., Leong Y. T., Nelson B. L., Jonathan W. C. Ng, and David S. L. (2005), Dispatching vehicles in a mega container terminal, $O R$ Spectrum, 27, 491-506.

Briskorn, D., Drexl A. and Hartmann S. (2006), Inventory-based dispatching of automated guided vehicles on container terminals, OR Spectrum, 28, 611630 .

Correa, A. I., Langevin A. and Rousseau L. M. (2007), Scheduling and routing of automated guided vehicles: A hybrid approach, Computers and Operations Research, 34, 1688-1707.

Egbelu, P. J. (1987), Pull versus push strategy for auto- mated guided vehicle load movement in a batch manufacturing system, J. Manufacturing Systems, 6, 209-221.

Evans, J. R. and Minieka E. (1992), Optimization Algorithms for Networks and Graphs, New York, Marcel Dekker.

Grunow, M., Guenther H. O., and Lehmann M. (2004), Dispatching multi-load AGVs in highly automated seaport container terminals, OR Spectrum, 26, 211235.

Grunow, M., Guenther H. O., and Lehmann M. (2006), Strategies for dispatching AGVs at automated seaport container terminals, OR Spectrum, 28, 587-610.

Kim, C. W., Tanchoco J. M. A., and Koo P. H. (1999), AGV dispatching based on workload balancing, International Journal of Production Research, 37, 4053-4066.

Lim, J. K., Kim K. H., Yoshimoto K., Lee J. H., and Takahashi T. (2003), A dispatching method for automated guided vehicles by using a bidding concept, OR Spectrum, 25, 25-44.

Morikawa, K., Takahashi K. (2009), A Flexible Branch and Bound Method for the Job Shop Scheduling Problem, Industrial Engineering and Management Systems, 8(4), 239-246.

Nguyen, V. D. and Kim K. H. (2009), A dispatching method for automated lifting vehicles in automated port container terminals, Computers and Industrial Engineering, 56, 1002-1020.

Nguyen, V. D. and Kim K. H. (2010), Minimizing empty trips of yard trucks in container terminals by dual cycle operations, Industrial Engineering and Management Systems, 9(1), 28-40.

Van der Meer, R. (2000), Operational control of internal transport, Ph. D. dissertation, Erasmus Research Institute of Management, Rotterdam, The Netherlands.

Vis, I. F. A. and Harika I. (2004), Comparison of vehicle types at an automated container terminal, OR Spectrum, 26, 117-143.

Yang, C. H., Choi Y. S., and Ha T. Y. (2004), Simulation-based performance evaluation of transport vehicles at automated container terminals, OR Spectrum, 26, 149-170. 